\title{
L'HOMME L'Homme
}

202 | 2012

Varia

\section{L'État, un ordre politique, un ordre cosmique}

Moogo de Michel Izard

\section{Alfred Adler}

\section{(2) OpenEdition}

\section{Journals}

Édition électronique

URL : http://journals.openedition.org/lhomme/23013

DOI : 10.4000/lhomme.23013

ISSN : 1953-8103

Éditeur

Éditions de l'EHESS

Édition imprimée

Date de publication : 4 juin 2012

Pagination : 15-51

ISSN : 0439-4216

\section{Référence électronique}

Alfred Adler, «L'État, un ordre politique, un ordre cosmique », L'Homme [En ligne], 202 | 2012, mis en ligne le 29 mai 2014, consulté le 30 avril 2019. URL : http://journals.openedition.org/lhomme/23013 DOl : 10.4000/lhomme.23013 


\title{
L'État, un ordre politique, un ordre cosmique
}

Moogo de Michel Izard

\author{
Alfred Adler
}

L'

ÉTUDE QU'ON VA LIRE était déjà envoyée à L'Homme lorsque nous avons appris le décès de Michel Izard. À ce qui, dans mon esprit, était un hommage à un ethnologue africaniste de tout premier plan, à un chercheur infatigable dont l'ouvre fait date dans l'histoire de notre discipline, s'ajoute maintenant un témoignage de profonde et fidèle amitié. Amitié pour un condisciple de jeunesse dont je partageais la passion pour les études de philosophie, amitié pour un collègue qui, tout au long de sa carrière professionnelle, a été un interlocuteur privilégié. Seule la mort a mis fin à un dialogue qui a toujours été pour moi source d'enrichissement intellectuel et de plaisir.

Michel Izard a consacré une longue partie de sa carrière scientifique à l'étude du Yatenga (Le Yatenga précolonial, 1985), l'un des deux plus importants parmi les royaumes mossi (ils sont au nombre d'une vingtaine) qui se sont constitués dans cette région de l'Afrique occidentale qui se fait appeler aujourd'hui Burkina Faso, nom choisi par le nouvel État pour remplacer celui de Haute-Volta porté à l'époque coloniale. Ses recherches avaient exigé des années de travail intensif sur le terrain et le dépouillement d'une somme considérable de documents d'archives et d'une littérature historique relativement abondante, comparée à d'autres aires culturelles du continent noir ; elles aboutirent à une thèse d'État monumentale soutenue en 1980. Celle-ci fit date dans le domaine africaniste et, au-delà, dans l'anthropologie politique, sous-discipline qui était encore en plein développement. Elle donna lieu à la publication de deux ouvrages : un gros volume à caractère essentiellement ethnologique (Gens du pouvoir, gens de la terre, 1985) et un autre de dimension plus modeste réservé aux données historiques. Vint ensuite un recueil d'articles (L'Odyssée du pouvoir. Un royaume africain: État, société, destin individuel, 1992) qui, s'ajoutant aux deux autres livres, parachevait non seulement la plus 
fouillée des monographies consacrées à l'étude d'un système politique africain, mais apportait déjà une contribution théorique décisive au problème général de la nature de l'État dans les sociétés traditionnelles. En 2003, Michel Izard publiait aux éditions Karthala un ouvrage qu'on peut considérer comme le véritable couronnement de son œuvre: il est intitulé tout simplement Moogo, mais le sous-titre qu'il lui avait donné, L'émergence d'un espace étatique ouest-africain au XVI siècle, indique l'ampleur de la vision à la fois historique et anthropologique qui était la sienne.

\section{Espace étatique, temporalité royale}

Mon propos est d'examiner ce qui, dans cette œuvre, touche aux fondements symboliques de l'institution de la royauté non seulement dans sa dimension spatiale que le titre choisi par Michel Izard met en avant d'entrée de jeu, mais dans sa dimension temporelle, l'une étant inséparable de l'autre. Et ce, sur les deux plans : celui de la structure interne de cette institution qu'il définit comme un État dont le concept est aujourd'hui universel et celui du contexte plus large, géographique et historique dans lequel s'inscrit cet État comme appartenant à l'ensemble moogo. Cet ensemble, l'auteur s'efforce de le saisir dans le passé en remontant aussi loin que la recherche des archives orales et des sources écrites permet de le faire et d'en suivre le développement jusqu'à la fin du XIX siècle, à la veille de la conquête coloniale. Certes, il est bien connu que l'émergence d'un ordre à proprement parler politique est conçue, depuis Henry J. S. Maine (Ancient Law, 1861) et Lewis H. Morgan (Ancient Society, 1877), comme le passage d'une organisation sociale fondée sur les liens de parenté (ou du sang, comme on disait communément) à une organisation où la primauté est accordée à ceux qui découlent de la cohabitation dans un même territoire. Un territoire qui, de ce fait, doit son identité à l'autorité sous laquelle il est placé et dont la légitimité est reconnue par tous ses occupants ou, à tout le moins, s'impose à tous quelle que soit la forme institutionnelle qu'elle revêt: instance réunissant les aînés de lignage et/ou des dignitaires détenteurs de fonctions rituelles éminentes (gérontocratie et/ou hiérocratie), chefferie (ou, pour reprendre le néologisme de Michel Izard, "cheffauté »), royauté, accompagnée ou non d'une administration à caractère étatique, expression qui reste encore à définir. Mais d'ores et déjà, on peut dire que si État il y a, l'autorité est dotée de prérogatives impliquant une certaine capacité d'exercer des contraintes sur la population aux fins de maintien de l'ordre, un ordre spécifique caractérisé par un ensemble de règles coutumières, lesquelles peuvent comprendre le recours, si besoin est, à la répression violente. 
J'ai rappelé cette distinction classique parce qu'elle donne sens à l'utilisation de la formule "émergence d'un espace étatique » retenue par l'auteur pour définir son projet, lequel dépasse le cadre d'une monographie, même si le Yatenga demeure sa référence essentielle au sein de l'espace qu'il désigne comme le Moogo (c'est-à-dire le "pays» mossi que l'auteur écrit "moose»). Un projet qui, au-delà même de cette vaste région d'Afrique occidentale, comporte une visée générale sur la nature de l'État que l'on peut qualifier à bon droit de philosophique. J'emploie ce qualificatif pour marquer toute la singularité de l'entreprise qui est celle de Michel Izard pour qui l'enquête de terrain la plus soucieuse (je dirais même la plus sourcilleuse) de procéder au recueil le plus complet possible des faits - sur les plans politique, social, économique et religieux -, observés jusque dans leurs moindres détails, ne se sépare pas d'une réflexion spéculative sur la nature de l'État. Cette singularité, qui tient sans aucun doute à la formation intellectuelle qu'il a reçue à l'université, le situe à distance des positions théoriques adoptées sur ce sujet par la plupart des anthropologues et plus particulièrement des auteurs anglosaxons (surtout les Britanniques). Ces derniers se disaient attachés à la conception et aux méthodes fonctionnalistes d'analyse des sociétés sans s'interdire pour autant - il s'agit des plus connus d'entre eux - de les combiner avec de larges emprunts à l'analyse structurale ${ }^{1}$, mais dans tous les cas, la référence à la philosophie était pour tous hors sujet dans le domaine des sciences sociales dont relève l'État, lequel n'est qu'une institution parmi d'autres.

C'est certainement l'un de leurs chefs de file reconnu comme le représentant éminent de ce courant, Alfred R. Radcliffe-Brown, qui exprime ce point de vue avec le plus de netteté dans la préface qu'il a donnée à African Political Systems (1964 [1940]). On en jugera par ces phrases si énergiquement martelées pour écarter du champ de notre discipline toute idée impliquant une "transcendance " de l'État, un mot qui porte le stigmate de l'esprit philosophique, attitude à bannir pour autant qu'on la veuille science sociale véritable, ce qui veut dire, selon lui, qu'elle doit prendre - autant que faire se peut - modèle sur les sciences de la vie. L'institution qu'est l'État ne saurait être appréhendée sous les espèces :

1. C'était, notamment, le cas dans le domaine africaniste de Jack Goody (tenté un moment par le marxisme) et de Siegfried F. Nadel (plutôt influencé par Max Weber), l'un des auteurs qui apporta une contribution à African Political Systems, l'ouvrage collectif dont nous parlerons plus loin. Mais l'appellation un peu lourdement académique de "fonctionnalo-structuralisme» s'appliquerait tout aussi bien aux positions théoriques de Alfred R. Radcliffe-Brown, le préfacier de cet ouvrage qu’à celles de ses « éditeurs », Edward E. Evans-Pritchard et Meyer Fortes. Le structuralisme dont il est ici question, faut-il le préciser, est fort éloigné de celui de Claude Lévi-Strauss. 
"[d'une] entité supérieure aux individus qui composent la société, [et] possédant comme l'un de ses attributs quelque chose appelée "souveraineté". On dit de l'État qu'il possède une volonté (le droit étant souvent défini comme la volonté de l'État) et émet des commandements. L'État dans ce sens n'existe pas dans le monde phénoménal, c'est une fiction de philosophe [...]. L'organisation politique de la société n'est que la partie de l'organisation sociale qui assume le contrôle et la réglementation du recours à la contrainte physique» (Radcliffe-Brown 1964 [1940] : XXIII).

Cette définition serait à peu près celle de Max Weber, à ceci près qu'elle est amputée d'une notion fondamentale, celle de légitimité (pour qualifier, notamment, la violence à laquelle l'État a recours quand il le juge nécessaire pour l'ordre social et sa propre sécurité, les deux choses allant de pair) dont on a peine à penser qu'elle puisse dispenser l'anthropologue ou le sociologue qui en fait usage de toute référence à une réflexion philosophique. Contrôle et réglementation sont des termes qui se rapportent plutôt à des pratiques qui conviennent mieux au fonctionnement des systèmes bureaucratiques qu'à celui des sociétés traditionnelles. On s'étonnera plus encore de ce dédain de la philosophie politique si l'on songe à la richesse, à cet égard, de celle qui nous a été léguée par les penseurs anglo-saxons, justement: de Hobbes à Locke et Hume, pour ne citer que les plus grands. Mais, en tout état de cause, peut-on parler de l'État en termes de fiction philosophique? Certainement pas si l'on prétend épingler ainsi un artéfact intellectuel fabriqué par de subtils manieurs d'abstractions qui feindraient d'ignorer l'histoire et la réalité politique de leur temps. Mais nous disons aussi que si l'on tient, non sans raison, à pointer un aspect fictionnel de l'État, ne serait-ce pas qu'il appartient en effet à l'essence même de cette institution dont la vocation est de faire du corps politique une totalité. Michel Izard nous parle bien $\mathrm{du}$ «fantasme unificateur de l'État».

\section{Qu'est-ce donc qu'un espace étatique?}

Concrètement, cette question porte sur une réalité physique et humaine dont l'étude constitue un préalable à celle du symbolisme spécifique qui accompagne ou signale le passage du non-État à l'État, passage progressif qui n'est jamais un saut d'un état (comme on parle en physique d'un état de la matière) à un autre. Pour Michel Izard, la tâche de l'anthropologie politique est de définir - toutes les données disponibles dans l'ordre historique, géographique, ainsi que linguistique ayant été prises en compte - un ensemble de conditions qui rendent possible l'apparition dans la zone concernée par ce passage d'une force conquérante (la notion de conquête sera discutée plus loin) mise en œuvre par un groupe particulier. Il ne peut s'agir, en effet, d'une population prise dans son ensemble 
(l'image d'un peuple en armes qui se mettrait en marche est tout à fait hors de propos, elle a même quelque chose de tout à fait fantaisiste), mais bien d'un groupe, pas forcément nombreux, d'individus plus ou moins en rupture de ban avec leur société et en possession d'une idéologie (on pourrait tout aussi bien parler d'une utopie) propre à être mise au service de la construction d'une société autre, d'un ordre nouveau. Serait-ce un État?

Nous pensons, et nous aurons aussi à y revenir, qu'on ne saurait faire de l'État comme tel la représentation d'une fin expressément recherchée par ceux qui en auront été, à vrai dire, les promoteurs à leur insu. Il n'y a pas, en effet, d'équivalence entre un changement, voire un bouleversement de l'ordre social existant - des changements et des bouleversements provoqués par les causes les plus diverses se sont produits tout au long de l'histoire des sociétés humaines - et l'émergence de l'État, et si vouloir l'un revient à créer l'autre, c'est tout le problème de l'anthropologie politique. Entendons d'une discipline - celle que le travail de Michel Izard nous aide à approfondir - qui ne saurait se contenter d'être descriptive et analytique mais s'attacherait à saisir les processus par lesquels sont advenues les différentes formes de pouvoir qui s'offrent à notre observation.

Il faut d'abord rappeler que le lien entre l'apparition de l'État (peu importe pour l'instant le contenu précis donné à ce terme) et l'arrivée d'un groupe conquérant a été affirmé par un grand nombre d'auteurs en quête "des origines de l'État", mais en négligeant ou en rejetant l'idée, véhiculée par les tenants de l'évolutionnisme (comme, par exemple, Morgan), qu'il serait lié à l'un des "stades avancés " de civilisation que le développement des sociétés assignerait aux institutions et, notamment, à celles qui touchent au pouvoir. Michel Izard s'efforce de traiter de la conquête comme d'un concept à élaborer, il nous parle "d'une problématique de la "conquête" ", terme que lui-même entoure de guillemets ${ }^{2}$. Il ne la considère pas seulement comme un fait, c'est-à-dire comme

2. Cela se comprend, notamment mais pas seulement, comme une mise en garde vis-à-vis des espèces d'images d'Épinal que l'enseignement de l'Histoire donné aux lycéens nous met dans la tête en privilégiant les grandes figures de conquérants, celles de l'Antiquité, celle de Napoléon et de bien d'autres. Figures de héros légendaires semi-mythisés, capitaines doués du génie des armes, à la tête de soldats vaillants, ils sont porteurs de projets grandioses et leur chute même n'entache guère la gloire que leurs exploits leur ont value. En Afrique, on a plutôt affaire à des héros fondateurs qui sont l'objet de récits mythiques. Seuls les conquérants musulmans qui ont combattu et soumis des populations au nom d'un jihad sont des personnages historiques dont nous avons connaissance par des chroniques rédigées en arabe ou en fulfulde écrit en caractères arabes. N'oublions pas de mentionner l'exception notable de Shaka, fondateur du royaume zulu (entre 1816 et 1818) et avec lequel des Européens furent en contact et purent recueillir de sa bouche quelquesunes de ses idées politiques et aussi ses questions sur l'organisation et le fonctionnement des armées du roi d'Angleterre. 
événement historique datable et dont il faudrait rechercher les causes particulières - ce qui est souvent, on y reviendra, un objectif hors de portée pour les africanistes, en particulier -, mais ce constat est certainement valable pour la plupart des ethnologues qui étudient les sociétés sans écriture. En ce qui concerne le Moogo, nous dirons que notre auteur adopte sur ce point une position extrêmement nuancée.

Il nous présente pourtant une très brève esquisse typologique: elle concerne la généalogie possible de deux sortes d'États. Sans doute le fait-il parce que la configuration des systèmes sociopolitiques de la région voltaïque semble y inviter : on y voit, en effet, se côtoyer et parfois même s'enchevêtrer des royaumes (petits ou grands), des chefferies, des sociétés lignagères ou des sociétés fondées sur la communauté villageoise, et le chercheur aimerait comprendre et débrouiller un tant soit peu le comment et le pourquoi d'une telle situation. C'est ainsi qu'il est amené à écrire que: "S'il faut faire initialement abstraction du fait étatique pour rechercher quelles sont ici les formes élémentaires de la vie politique, nous constatons qu'il y a coexistence de deux grandes traditions: celle qui privilégie la communauté villageoise au détriment du lignage, et celle qui propose la formule inverse " (Izard 2003 : 62).

Observant qu'à ces deux formes peuvent correspondre des sociétés sans État (dont le caractère distinctif est l'absence de toute centralisation du pouvoir) aussi bien que des sociétés qui en sont pourvues (royautés, chefferies comportant une forte concentration du pouvoir) ${ }^{3}$, il affirme à propos de celles-ci, que la communauté villageoise conduit à «l'État-cité, dont la territorialité, par définition, est centrée, et l'autre, parallèlement, conduit de l'organisation lignagère à ce qu'il est commode d'appeler l'État-territoire, dont la territorialité est acentrée » (Ibid.). La connotation causale que pourrait comporter le verbe "conduire» est immédiatement corrigée, si je puis dire, par la négation du caractère inéluctable du passage

3. Une question se pose : pouvons-nous alors avoir affaire à des formes élémentaires au même sens du terme dans l'un et l'autre cas? Peut-on dire que s'interroger sur les formes élémentaires de l'État revient à s'interroger sur les formes élémentaires de la vie politique en général, y compris dans les sociétés sans État? Manifestement, Michel Izard laisse ces problèmes en suspens mais sa position a le mérite, à mon sens, de refuser d'essentialiser la dichotomie société sans État/société avec État que beaucoup d'anthropologues avaient acceptée comme allant de soi ou en émettant des réserves mais sans rien proposer à la place. Une exception notable, mais restée sans suite dans les études africaines, est la contribution de Robin Horton à The History of West Africa (1971). Ce texte intitulé "Stateless Societies in the History of West Africa" analyse le rôle politique déterminant des associations (secrètes ou non) et des classes d'âge dans les populations du Sud-Est du Nigeria (région de la Cross River) et, en dépit de son titre, fait la démonstration de l'importance des institutions intermédiaires entre celles propres aux systèmes étatiques et celles qui appartiennent à des systèmes où la forme étatique est demeurée, si l'on peut dire, latente. J'observe que Horton retrouvait ainsi une des idées maîtresses que Robert Lowie avait développée dans The Origin of the State, ouvrage publié en 1927. 
du non-État à l'État. Et pour cause! Dans cette aire culturelle voltaïque où ont pris naissance les divers royaumes moose, à l'extérieur comme à l'intérieur de l'espace du Moogo, de multiples sociétés non étatiques ont persévéré dans l'existence des siècles durant. Ce qui suppose, note Michel Izard - et ce que vérifient les traditions orales -, que lorsque la situation l'exigeait (par exemple, dans le cas des expéditions guerrières que les rois entreprenaient généralement pour faire des captifs dont le rôle était décisif dans le fonctionnement de la machine étatique), surgissait dans ces sociétés " une dynamique anti-étatique ». Et en effet, celles-ci étaient non seulement capables d'organiser leur défense mais aussi de passer avec succès à l'offensive. Les remarques qui font suite à ce constat vont même plus loin.

Le parallélisme entre les processus qui aboutissent aux deux formes d'État indiquées est mis en cause par notre auteur qui voit d'importantes différences entre les principales aires culturelles voltaïques et observe que des deux séquences retenues par sa typologie c'est seulement la séquence organisation lignagère-État-territoire qui de fait s'est déroulée dans l'espace moogo ou, plus précisément, "moore-dagbane » auquel il adjoint avec raison l'espace "gulma ", c'est-à-dire le pays gourmantché. Michel Izard évoque alors ce qu'il appelle " un moment capital de l'histoire de l'anthropologie politique africaniste». Assurément, car ce moment n'est autre que celui de la parution en 1940 de African Political Systems, ouvrage déjà mentionné plus haut et dont les éditeurs intellectuels furent Edward E. Evans-Pritchard et Meyer Fortes. Il se trouve que les contributions de ces deux maîtres de l'anthropologie britannique sont consacrées à des systèmes politiques qui reposent l'un et l'autre sur des structures lignagères qui sont fort différentes et qui en représentent, selon notre auteur, deux modèles majeurs. Le modèle nuer étudié par Evans-Pritchard dans le Sud-Soudan, est celui du lignage segmentaire dont lui-même a pu dire qu'il correspondait à un système politique qui « loin d'être chaotique [...] revêt une forme stable et cohérente que l'on pourrait appeler une "anarchie ordonnée" "(1964 [1940] : 256). Le cas des Talensi (une population voltaïque du Nord-Ghana) étudiés par Fortes (1964 [1940]) est bien sûr celui qui retient l'attention de Michel Izard qui écrit ceci :

«Pour l'essentiel, il apparait que le système lignager talensi [...] est aussi celui des sociétés globales étatiques voisines des royaumes nord-ghanéens et du Moogo. Tout se passe comme si les Tallensi $[s i c]$ et leurs proches voisins non étatiques fournissaient l'exemple de ce qu'étaient les sociétés "moore-dagbane" avant que le processus de centralisation du pouvoir ne se développe dans la région et au-delà " (2003: 63).

Ainsi y a-t-il passage du non-État à l'État et coexistence de l'une et l'autre forme d'organisation politique. Nulle nécessité historique ne commande ce passage. CQFD. 
Michel Izard poursuit ainsi ce propos :

«À cet égard, l'exemple des Talensi est éloquent. En effet, pour pouvoir traiter cette société en objet ethnographique autonome, Fortes, méthodologiquement à juste titre, est conduit à oblitérer dans une large mesure le fait que l'espace social des Talensi est intégré [c'est moi qui souligne] à l'espace politique des Mamprusi, où nous avons bien cette coexistence du non-État et de l'État évoquée plus haut, que l'on peut retrouver à l'identique sur l'ensemble des territoires étatiques que nous considérons, et singulièrement du territoire du Moogo ».

Il n'est pas inutile de s'attarder ici un moment pour s'interroger sur ce "moment capital» de l'histoire de notre discipline tel que l'envisage Michel Izard. Nous avons vu qu'après avoir distingué deux processus selon lesquels s'effectue le passage du non-État à l'État, il repère deux modèles d'organisation lignagère dont l'un, celui qui prévaut chez les Talensi, une population appartenant comme les Moose à l'aire voltaïque, nous permettrait de comprendre non seulement la coexistence du non-État et de l'État mais l'intégration, "dans une certaine mesure", du premier dans le second, c'est-à-dire de son «espace social» dans celui de l'État, l'État mamprusi, en l'occurrence. Ainsi les structures étatiques seraient en mesure d'inclure en elles et pas seulement de coexister avec elles, des structures encore purement lignagères et claniques, des témoins vivants et non des témoins au sens archéologique de ce terme, d'un état antérieur pré- ou proto-étatique. Meyer Fortes aurait avec raison "oblitéré » ce fait afin de donner à sa recherche "un objet ethnographique autonome". Faudrait-il comprendre par là que l'objet ethnographique en question serait susceptible d'être appréhendé dans sa véritable nature, comme un système politique non déformé, non "contaminé" par le caractère étatique de l'ensemble englobant? Le lecteur peut ne pas être au fait (ou est-il supposé en savoir assez tant le cas nuer est connu ?) de ce qui définit la spécificité du modèle nuer et donc pourquoi et comment il est en opposition décisive avec le modèle talensi. Il peut également n'être guère plus éclairé sur ce dernier mais néanmoins prêt à admettre que son appartenance à la même aire culturelle et au même large espace que les Moose serait propice à une meilleure appréhension du processus de formation des États de l'espace moogo. Or, la contribution de Meyer Fortes à African Political Systems ne néglige pas, me semble-t-il, les rapports entre les Talensi et les Mamprusi et cela d'autant moins que les Talensi sont une société composite : avec, d'une part, ceux qui portent cet ethnonyme, les gens de la terre (ou descendus du ciel) dont les aînés des lignages maximaux fournissent les tendaana, les chefs ou les prêtres de la terre (teng), puissance qui est l'objet d'un culte constituant un élément essentiel de leur système religieux et, d'autre part, des clans immigrés, les Namoos, 
descendants des Mamprusi et détenteurs de la chefferie. Un mythe d'origine raconte l'arrivée dans le pays talensi de leur ancêtre nommé Mosuor et explique comment il a pris possession de sa chefferie pour avoir, notamment, par son curieux couvre-chef rouge, inspiré une grande peur aux autochtones. Cette différence de statut est allée de pair avec une stricte complémentarité rituelle entre les deux formes d'autorité indissociables l'une de l'autre et qui constituent "deux pôles opposés se contenant mutuellement" (Fortes 1964 [1940] : 223) ${ }^{4}$. On ne saurait donc parler d'intégration même purement symbolique de l'espace social talensi (Talensi et Namoos) dans l'espace politique du royaume mamprusi dont le caractère étatique, au demeurant, est nettement moins marqué que dans le Yatenga, par exemple, à en juger, précisément, par l'analyse qu'en a faite Michel Izard.

S'il fallait comparer les systèmes politiques nuer et talensi - l'un et l'autre lignagers - tels qu'ils sont décrits par Evans-Pritchard et Fortes respectivement, on pourrait dire que dans ces deux sociétés par ailleurs si différentes, mais où les rapports entre les clans et/ou les lignages sont fondamentalement égaux d'un point de vue économique comme politique, il existe un clivage qui est assurément politique, néanmoins, il n'a d'autre portée que symbolique, ce qui ne signifie pas qu'il soit de peu de réalité. Ainsi, Evans-Pritchard appelle aristocrates les Nuer qui appartiennent au clan ou au lignage principal qui occupe une position dominante dans la tribu dont il constitue un segment. Cette supériorité est liée au fait que la tribu comme telle est une entité territoriale permanente fondée sur la prééminence d'un clan en charge des rituels de propitiation. Les lignages, quant à eux, sont très mobiles ${ }^{5}$. Mais hors de son territoire tribal, un "aristocrate» n'est en rien supérieur à qui que ce soit.

Regardons maintenant du côté talensi. Les Namoos détenteurs de la chefferie peuvent bien se dire nobles parce qu'ils sont les descendants, mais pas tous, bien sûr, de Mosuor, un membre du lignage royal mamprusi qui, ne réussissant pas à accéder au pouvoir dans son territoire natal, fut obligé de prendre la fuite. Il est intéressant de noter que les deux clivages sont de sens inverse. C'est une forme d'autochtonie ou plutôt

4. Une phrase de Fortes condense les aspects essentiels de cette complémentarité : «Le mécanisme le plus manifeste qui, pour le bien commun, permet de maintenir l'interdépendance rituelle et la responsabilité solidaire des chefs et des tendaana est le cycle des grands festivals » (1964 [1940] : 226). Le même auteur avait consacré à l'étude détaillée de ce cycle un admirable article qui en fait la démonstration (cf. Fortes 1936).

5. Il faut préciser que les Nuer sont une société pastorale qui fut, au XIXe siècle, une «nation » (Evans-Pritchard emploie ce terme qui peut nous étonner) conquérante qui a intégré en son sein nombre de leurs voisins Dinka capturés lors des guerres fréquentes où s'affrontaient ces deux populations de pasteurs. 
sa position de premier occupant d'un territoire tribal qui, chez les pasteurs nuer, confere aux membres de ce clan une très relative supériorité. Pour les cultivateurs talensi, une supériorité tout aussi relative est conférée aux membres du lignage issu de Mosuor, lequel est un étranger pour eux, mais un étranger auquel ils reconnaissent certains pouvoirs cachés (ou "mystiques ", comme l'écrit Fortes) qu’ils lui ont "achetés " en lui offrant à titre de contrepartie un droit d'installation chez eux. Ces deux systèmes politiques fondés sur deux formes très différentes de l'organisation lignagère sont, selon la perspective adoptée sur la direction politique qu'elles auraient tendance à prendre - il s'agit là d'une pure hypothèse d'école - aussi proches ou aussi éloignés d'une structure, à proprement parler, étatique, c'est-à-dire et nous y insistons, telle qu'elle est décrite et analysée par Michel Izard. En définitive, le recours qu'il fait à cette typologie pour expliquer la genèse des formes étatiques me semble, en l'occurrence, poser plus de problèmes qu'elle ne permet d'en résoudre. Revenons à la notion de conquête.

\section{"Conquête ou mutation endogène?"}

C'est le titre très parlant d'un paragraphe de Moogo. Il n'y a pas de réponse tranchée par oui ou par non. La démarche de Michel Izard l'amène à un changement de registre, il passe de celui de la morphologie sociale à celui de l'essence d'un système politique. Lorsqu'il s'agit de l'édification d'un État, nous avons affaire, selon ses termes, à " une œuvre de pensée»; cette proposition vaut pour l'Afrique précoloniale et pas seulement pour l'Occident ou l'Orient ancien et moderne et, de manière générale, pour les civilisations en possession de l'écriture et donc dotées d'une classe de scribes et de doctes (prêtres et hauts dignitaires de toutes sortes) à même de la concevoir et de la théoriser ${ }^{6}$. Il faut assurément, si elle doit se mettre en mouvement et produire des effets, que cette " œuvre

6. Dans un article consacré à Moogo, le chercheur belge Mathieu Hilgers (2009) s'interroge précisément sur cette difficulté que rencontre l'ethnologue soucieux d'analyser " une œuvre de pensée " telle que l'énonce Michel Izard en l'absence de texte qui permette d'accéder " aux intentions " des acteurs historiques à l'origine de la création de l'État. Ce chercheur considère qu'expliquer l'action politique, y compris dans les sociétés sans écriture, suppose la connaissance des intentions explicites de ceux qui la conduisent et que la pensée symbolique, celle qui s'exprime dans les mythes et le rituel, ne saurait en rendre compte. À s'y tenir, on ne ferait que creuser plus profond le fossé séparant "primitifs " et " civilisés " et donc méconnaître, en l'occurrence, l'universalité du politique comme dimension de la condition humaine. Hilgers, malgré de curieuses réserves motivées par l'absence de référence "aux protestations populaires qui visent le pouvoir et la cour", rend hommage à Michel Izard d'avoir échappé à ce travers. Je suis d'accord, mais pour autant, je ne crois pas que la notion d'intention dans sa généralité psychologique soit utile à l'anthropologue qui ne saurait qu'en inférer la présence dans l'esprit de ceux qui agissent. 
de pensée" s'avère capable d'attirer à elle et de concentrer pour la développer, une certaine puissance matérielle, en l'occurrence, un groupe de personnes unies par des intérêts factionnels communs et qui se constitue donc en tant que dissidence ne pouvant mener qu'à la rupture avec l'ordre existant. Il est possible - les exemples ne manquent pas que cette force trouve, sans qu'il soit toujours nécessaire de supposer d'imposants déploiements militaires, car souvent la ruse joue un rôle décisif (le thème de la ruse pour s'emparer du pouvoir, rappelons-le au passage, est présent dans de nombreux mythes de fondation des royaumes africains), un terrain favorable pour se répandre et s'imposer à des populations qui accepteront la domination des nouveaux venus avec des réactions variables. Celles-ci peuvent aller de la résistance, passive ou active, à la résignation, voire à l'adhésion aux valeurs des vainqueurs, ce qui finit toujours par se produire; une conquête n'est pas une occupation ou du moins, si elle peut l'être un certain temps, cela ne saurait durer. Même si la forme de domination qui s'est alors constituée a tendance, dirons-nous, "naturellement" à croître, tôt ou tard elle sera forcément limitée par une puissance égale ou supérieure. Un point essentiel de l'analyse qui nous est proposée est que la force qui met une limite, voire dresse une véritable barrière défensive à laquelle vient se heurter le groupe conquérant, n'est pas dans chacun des cas effectivement observés, un autre État travaillé lui aussi par les mêmes tendances à l'expansion. Michel Izard cite maintes situations où s'est manifestée la résistance tenace - et, en définitive, victorieuse - de sociétés villageoises ${ }^{7}$ coalisées ; parfois celles-ci tirent leur force de leur invocation d'une origine commune plus ou moins légendaire, car faisant référence à un héros dont les tenants de la tradition affirment l'existence historique. Nombreux sont les cas où, malgré l'absence de toute autorité centralisée, ces sociétés sont unies transversalement par des alliances matrimoniales et/ou par des liens rituels, lesquels peuvent se manifester, par exemple, dans l'enchaînement ordonné des fêtes de leur calendrier saisonnier ou encore dans le temps des sorties de leurs masques ou de leurs sessions initiatiques respectives ${ }^{8}$. Elles apparaissent comme

7. Sur le plan strictement politique, la notion de société villageoise appliquée à l'Afrique noire mériterait de longs développements qui ne trouvent pas place ici. Selon que l'on a affaire à un habitat concentré ou dispersé, à une société pastorale, agro-pastorale ou presque exclusivement agricole (alors la variable est celle des méthodes culturales - intensives et/ou extensives), la forme politique que prend l'organisation de la communauté locale va présenter d'importantes différences.

8. Des recherches (il s’agit dans la plupart des cas de thèses qui, à ma connaissance, n'ont pas été publiées) sur la diffusion des cultes initiatiques en Afrique occidentale, dans les aires voltaïque et mande, ont permis de mettre en lumière de véritables "sociétés internationales" comme celles du Komo, du Bagre, du Jo (joro en pays lobi) ou du poro (en pays sénoufo et chez les Mande sud de Guinée, Sierra Leone et Liberia), pour ne citer que les plus connues, dont le rôle .../... 
des enclaves d'autonomie politique au milieu d'États aux limites mal définies et leur perpétuation au cours des siècles nous donne à penser que les bases sur lesquelles elles étaient assises n'étaient pas plus fragiles que celles des États. Le mot d'enclave ne convient pas toujours pour définir ces espaces non étatiques ainsi que les relations qu'ils pouvaient avoir avec les États, qu'elles fussent positives (pacifiques et faites d'échanges commerciaux ou même de services rituels) ou négatives (guerrières et faites de pillages). Nous n'avons pas ici affaire à une différence de nature entre des territoires qui seraient séparés par des barrières plus ou moins étanches. Le concept de frontière n'est nullement absent de la réalité géopolitique de l'Afrique précoloniale mais, comme on peut s'y attendre, il ne correspond pas à un tracé linéaire fixé et accepté par les autorités en place de chacun des côtés ainsi séparés. S’il fallait lui trouver, de façon toute provisoire, une définition moins arbitraire, je dirais qu'on a affaire, en l'occurrence (et cela ne concerne pas seulement cette région de l'Afrique), à une frontière "épaisse", selon le qualificatif proposé par le linguiste et mathématicien Jean-Pierre Desclés (2011) pour décrire la relation de voisinage entre des territoires qui tout en étant bornés (il y a un dedans et un dehors) s'interpénètrent jusqu'à un certain point: la limite de l'un est à l'intérieur de l'autre et réciproquement. Autrement dit, cette limite qui les sépare n'est pas sans dimension.

Dans un texte intitulé "La politique extérieure d'un royaume africain ", Michel Izard (1982) nous apprend que :

" [...] pendant des siècles, la frontière séparant le Yatenga des royaumes-tampons de Yako et de Tema, délimitera deux mondes moose étrangers et potentiellement hostiles l'un à l'autre'. Nettement tracée, s'appuyant toujours sur des repères naturels (lits de marigots), elle sert d'axe à une assez large bande de terres inhabitées : les premiers villages, de part et d'autre de la frontière, en sont éloignés de plusieurs kilomètres; ce sont pourtant des villages exposés où, au XIXe siècle, on plaçait plus volontiers des chefs "roturiers" que des princes».

L'auteur poursuit son analyse en observant qu'en contraste avec les relations entre États en tant que "souverains" politiques, les relations commerciales ignorent les frontières formelles que ceux-ci se donnent et qui ne concernent ni les caravaniers qui opèrent sur d'immenses espaces

[Suite de la note 8] politique a été considérable, qu'il ait été en rapport de complémentarité ou d'opposition avec celui des chefferies, là où elles existaient. Quoi qu'il en soit, ces "Internationales" avaient juridiction (politique et parfois même économique) sur de vastes territoires, elles avaient aussi les moyens matériels et spirituels (notamment, les sorties de masques, le recours à la magie) de faire respecter leurs «lois » et de sanctionner durement les récalcitrants.

9. Il s'agit, bien entendu, des deux principaux royaumes du Moogo : le Yatenga et le Woagdogo. Cette capitale royale a été appelée Ouagadougou par les Français, nom conservé par la capitale du Burkina Faso. 
ni les "gens de la terre", les forgerons, en particulier, qui offrent leurs services là où l'on a besoin d'eux. À l'ouest du Yatenga, nous trouvons le pays samo (un groupe de langue mande) "dont les habitants sont organisés en grosses communautés villageoises indépendantes » Celles-ci résistèrent toujours avec succès aux assauts répétés des cavaliers moose résignés à constater : "que ces paysans sans chefs étaient de redoutables guerriers" (ibid. : 367). Ainsi, ces frontières " épaisses » dont nous parlions (et peut-on imaginer des frontières plus « épaisses » que ces espaces plutôt restreints et à l'autonomie toute relative qu'il désigne comme des « royaumestampons" ?) sont, ou furent, des frontières mouvantes, dynamiques qui nous donnent à penser le caractère de précarité, dans l'espace et dans le temps, de l'emprise des conquêtes attestées dans cette partie de l'Afrique.

Sans l'affirmer de façon tout à fait explicite, Michel Izard laisse entendre qu'en définitive, les véritables frontières sont d'ordre rituel ou, plus exactement, politico-rituel. Il décrit, en effet, en ces termes la situation qui fait suite - et le plus souvent met fin - à la période d'incertitude et de précarité qui accompagne toute conquête :

"À l'intérieur d'un territoire dont la stabilité des frontières indique que le temps des conquêtes est terminé, et sur lequel l'État prend forme et puissance de s'être assuré, de gré ou de force, la légitimation par l'onction de la terre [c'est moi qui souligne], les conquérants peuvent bien conserver leurs armes, ils n'ont cependant plus d'ennemis à combattre" (1992: 43).

Si nous comprenons bien, cela signifie que comme le veut la logique de l'affrontement entre unités locales voisines (au sein de l'espace du Moogo ou dans l'espace environnant), qu'elles soient étatiques ou non, la frontière, en donnant alors à ce mot un sens strictement politique, est la délimitation qui résulte d'un processus de pacification arrivé à son terme et qui a donc abouti à la reconnaissance mutuelle du territoire respectif que chacune occupe dès lors de plein droit. Sont ainsi créées les conditions d'une paix plus ou moins durable, mais la limite formellement admise et consacrée, celle pour laquelle on ne se fait pas la guerre et qui fait sens pour les habitants («les gens de la terre») c'est celle d'une unité de maitrise de la terre, portion du territoire et, en l'occurrence, division du royaume ${ }^{10}$, au bénéfice de laquelle le responsable du culte des autels de la terre, tenga, exerce son office.

10. L'analyse de ces unités de maîtrise de la terre est présentée par Michel Izard dans Gens $d u$ pouvoir, gens de la terre (1985a). Indiquons seulement que ces unités territoriales ne coïncident pas toujours, il s'en faut, avec les unités " administratives " de commandement, c'est-à-dire celles qui sont placées sous l'autorité directe du pouvoir central. Elles correspondent en fait aux divisions de l'espace pré-moose et donc à des populations ethniquement et culturellement hétérogènes. Leur intégration au sein du système politique unitaire du royaume du Yatenga n'a pas effacé toute marque de leur identité historique. 
En pays moogo, l'espace étatique se caractérise par l'existence d'un centre, c'est le village royal (na-tenga) dont l'emplacement change à chaque nouveau règne; plus on s'en éloigne en direction de la périphérie, c'est-à-dire en allant au-delà des localités excentrées encore sous le commandement de dépositaires de l'autorité du naam (c'est-à-dire des nakombse, les chefs qui possèdent cette substance du pouvoir dont nous préciserons plus loin la nature), plus on risque de se retrouver dans un no man's land. Et dans ces marges de l'État, il se passe pas mal de choses: y sévissent, en particulier, des bandes de pillards qui, le plus souvent, viennent du dedans.

L'article intitulé "La lance et les guenilles " ${ }^{11}$ décrit avec des formules fortes et parfois même percutantes, la dérive de ces princes déchus - leur titre est purement nominal - transformés en guerriers dépenaillés et sans cause, en "gens sans foi ni loi ». Leur situation misérable résulte de leur position généalogique marginale dans le moos buudu (c'est-à-dire le lignage royal strictement patrilinéaire) qui les écarte automatiquement du droit à la succession à la fonction de chef à laquelle n'accède que celui dont le père a été lui-même chef et donc fils de chef. Ces fils perdus ne sont pas seulement hors du temps, celui de l'État, un temps, précisément, qui n'est plus celui des commencements, celui des conquérants (une fois institué, l'État s'affirme comme ayant été déjà là depuis les origines), ils sont aussi :

« Hors de l'espace : car l'espace royal comme l'espace villageois est interdit aux nakombse. Rejetés hors des villages, les nakombse ne trouvent pas pour autant place dans les lieux du pouvoir central, où le roi s'entoure de serviteurs pour mieux tenir ses parents à l'écart, la fidélité de ceux-ci étant beaucoup moins assurée que la loyauté de ceux-là. Craints, méprisés aussi, [ils] mènent une existence marginale vouée à la guerre et au pillage [...]. Dureté, morgue, démarche lente de cavalier fatigué : des nakombse, qu'on ne distingue autrement des gens du commun que par leur scarification faciale surnuméraire et leur bonnet » (Izard 1992: 52).

En retraçant les grandes lignes de la formation du royaume du Yatenga, Michel Izard en vient à mettre en évidence les deux processus fondamentaux, à la fois opposés et complémentaires, qui conduisent à l'établissement d'un système étatique centralisé: d'une part, la marginalisation des nakombse, dont il vient d'être question et, d'autre part, la disposition selon laquelle est réservé aux captifs royaux seuls l'accès aux plus hautes fonctions de service et d'autorité, dotant ainsi le détenteur du pouvoir suprême du moyen de l'exercer sans partage, sans subir de pression de mentionné L’Odyssée du pouvoir (1992). 
la part de rivaux potentiels. Mais cette marche vers la concentration du pouvoir entre les mains d'un souverain n'a rien d'un mouvement uniformément déterminé et qui ne rencontrerait aucun obstacle. Comme l'écrit Michel Izard : "Si l'État centralisé ne peut prendre définitivement forme qu'à partir du moment où s'est engagé un processus irréversible de désegmentarisation de la société (la segmentarité, ici, est à la fois lignagère et territoriale), la constitution d'un appareil d'État dont les agents sont pris au sein de la société segmentaire ne laisse pas d'introduire dans la mise en œuvre du projet despotique une difficulté quasi insurmontable " (Ibid.: 53). Difficulté qui se résume en ceci que la solidarité entre membres d'un même lignage résiste à l'éloignement de certains d'entre eux et que le système ne peut pleinement se développer qu'avec l'apparition "d'une spécialisation familiale dans le service de Cour, de la formation d'une "caste" de grands officiers de l'État".

Le terme de caste me semble, en l'occurrence, d'un emploi judicieux car il caractérise bien la nature de l'une des transformations sociales qui accompagnent l'étatisation de la fonction politique du roi. Ce dernier doit à la possession du naam la sacralisation de sa personne et de son autorité, mais nous examinons ici les moyens dont il dispose pour exercer le pouvoir suprême - «il est seul et règne seul sur son peuple ». Le système y pourvoit en l'entourant de captifs royaux, ces "gens la force" (panga, notion opposée à celle de naam) que l'on dépouille des marques essentielles de leur identité : ils sont désocialisés, ils perdent leur nom, ils sont rasés; intégrés dans le groupe de descendance maximale des Moose, ils s'appellent tous Wedraogo, le nom patronymique de leurs maîtres. Ils deviennent gens de caste (ou quasi-caste) en ce sens qu'ils sont définis par leur fonction qui consiste dans les tâches qui sont les leurs: pages, palefreniers, gardes du palais, travailleurs sur les champs royaux. Ils ont aussi et peut-être surtout le rôle d'une police car eux seuls peuvent, au nom du roi, arrêter, emprisonner, spolier et tuer des gens. Ils ont une loi : obéir aux ordres de leur maître, quant aux règles de l'honneur, elles ne valent pas pour eux car ils abandonnent toute éthique lignagère. C'est ainsi que dans un royaume traditionnel africain prend naissance ce qu'on peut, à bon droit, désigner comme un appareil d'État. Mais il ne faut pas oublier l'autre face, la face sombre de leur service, celle qui comporte la garde des regalia et de diverses reliques et insignes royaux, c'est-à-dire des symboles puissants et menaçants de la sacralité du pouvoir. Ils s'occupent aussi des funérailles des épouses royales et enfin, ce sont eux qui, à la nomination d'un nouveau roi, mettent à mort le cheval sacré de son prédécesseur. Deux fortes formules qui condensent le double aspect de ce type si spécifiquement africain d'appareil de pouvoir et dont nous 
pouvons maintenant saisir toute la portée viennent sous la plume de Michel Izard: " [...] le despotisme est la négation de la segmentarité » (1992: 54) et "Les captifs royaux échangent leur vie contre la souillure despotique" (ibid. : 55).

Si le propre du despotisme est de se vouloir sans limite, c'est dans l'emprise sur les hommes qu'il s'exerce, l'expansion de l'État est, quant à elle, nécessairement soumise à des limitations de toutes sortes. Des limitations plus ou moins strictes qui sont à mettre au compte de facteurs divers : géographiques, économiques, culturels ou autres, sans oublier le rôle des technologies dans lesquelles sont incluses les méthodes de combat liées aux armes utilisées par les fantassins et à l'existence ou non de groupes de cavaliers, tous facteurs dont l'anthropologue s'efforcera d'évaluer le poids respectif. Il ne saurait, en effet, se contenter d'invoquer la contingence de l'événement. Il s'agit au demeurant d'une double contingence, celle du temps et des circonstances de la naissance de l'État (déterminables à condition que le chercheur puisse au moins établir une chronologie raisonnée des règnes, pour autant que les traditions orales le permettent), celle de son déclin, voire de son effondrement dont il aura à rechercher les causes qui peuvent rester tout aussi obscures que celles qui ont concouru à sa naissance.

Mais la force, qu'elle soit conquérante (au sens de conquête territoriale suivie de la soumission totale ou partielle de la population autochtone à l'ordre nouveau) ou seulement partie prenante (par l'action de petits groupes étrangers disposant d'une supériorité reconnue par les "conquis") dans un processus sociopolitique aboutissant à l'émergence dans un espace jusque-là non étatique de l'institution surimposée qu'est l'État, ne peut rien sans ce que Michel Izard, on vient de le voir, nous a désigné comme une "œuvre de pensée». C'est le lieu de citer la forte formule qui émaille, parmi d'autres formules tout aussi tranchantes, les pages finales de Moogo: "À la naissance de l'État, il y a une contingente révolution technique et une nécessaire révolution intellectuelle " (2003: 370).

Touchant aux conditions de possibilité de l'apparition de l'État, c'est donc ainsi que Michel Izard conçoit la différence de nature entre les forces matérielles qui sont à réunir et la force "spirituelle » qui est à mobiliser. Nous sommes fondés, en l'occurrence, à reprendre le terme utilisé plus haut d'idéologie, un concept dont je dirais qu'il doit être pris plutôt dans l'usage qu'en a fait Georges Dumézil ${ }^{12}$ que les auteurs qui se réclament

12. Michel Izard s’y était référé explicitement dans un article de 1995 intitulé : «Une trifonctionnalité africaine ? Représentations de la souveraineté et catégories socio-fonctionnelles dans le Yatenga ». 
du marxisme. Une idéologie, en effet, dans laquelle se combinent, d'une part, des thèmes mythologiques ou légendaires appartenant à un fonds ancien et que peuvent partager de nombreuses populations voisines ou même établies en des lieux fort éloignés du Moogo ${ }^{13}$ et, d'autre part, des principes proprement politiques mais enveloppés dans la substance mythique et fournissant les fondements de l'organisation du pouvoir (du centre à la périphérie du territoire) et des justifications quant aux moyens de l'exercer en recourant à la coercition en cas de nécessité.

De manière tout à fait significative, le chapitre premier de Moogo et qui fait suite à une très longue et substantielle introduction, a pour titre : "Une société pour l'État». L'allusion au titre de l'ouvrage de Pierre Clastres est transparente: comme si, à la notion aussi provocante que contestable à ses yeux de "société contre l'État ", il lui fallait opposer une formule contraire, c'est-à-dire qui exprime, mais en inversant le rapport entre les termes, un même point de vue finaliste sur la question des relations entre société et État ${ }^{14}$. Une question peut-être naïve se pose: comment une société peut-elle être contre ou pour l'État?

Une première remarque de simple logique s'impose, c'est qu'il y a implication mutuelle. La coexistence, la simultanéité au moins virtuelle de chacun des deux termes est nécessaire sinon comment feraient-ils pour se rencontrer et, éventuellement, se séparer. Car si une société étatique peut intégrer une ou plusieurs sociétés non étatiques, ces dernières peuvent, si elles n’ont pas été complètement ou trop fortement déstructurées, revenir à un (ou au) mode de fonctionnement politique qui devient le leur ou était le leur, à l'occasion d'un affaiblissement ou, à plus forte raison, de l'effondrement de l'État dont elles ont fait partie. Les exemples ne manquent pas en Afrique mais aussi dans l'Amérique précolombienne. À Clastres, on n'a pas manqué de demander comment une communauté

13. Après avoir rappelé que cette espèce de révolution que présuppose l'apparition de l'État s'inscrit en réalité dans la longue durée, Michel Izard écrit à propos des lointaines origines de l'espace politique du Moogo que «la forme politique que nous identifions à l'État a été familière à de vastes régions de l'Ouest africain, sans doute dès la plus haute Antiquité, après quoi les grands empires sahélo-soudaniens ont constitué un gisement particulièrement riche de représentations et d'expérimentations dans lequel il a été loisible à beaucoup de sociétés de puiser ce qu'elles voulaient, de solliciter pour la poursuite de leurs fins propres un immense savoir continûment transmis et sans cesse transformé" (2003: 146).

14. Si la notion de finalité (et corrélativement d'instrumentalité) peut ici surprendre, songeons au fait que, s'il est concevable que l'État soit pour une société qui est la réalité première un moyen qu'elle adopte et adapte en vue des fins qui sont les siennes, il l'est tout autant que la société devienne sinon un moyen du moins un objet, un matériau que l'État modèle en vue des fins que lui seul s'autorise à définir et qu'il cherche à atteindre quel qu'en soit le prix, s'il le juge nécessaire. L'histoire de l'humanité ne manque pas hélas d'exemples de ces systèmes, proches ou lointains, illustrant ce que nous avons désigné au $\mathrm{XX}^{\mathrm{e}}$ siècle qui a été, si l'on ose dire, son âge d'or, du nom de totalitarisme. 
«indivise» et n'admettant aucune inégalité entre ses membres, pourrait avoir la moindre expérience ou même une quelconque idée de la menace représentée par une institution qu'elle est censée seulement pressentir plus ou moins confusément (encore faudrait-il analyser sinon les causes, du moins les symptômes d'un tel pressentiment) sinon les ignorer. Le temps ne lui a pas été laissé pour répondre et sans doute aurait-il su prendre en compte les importantes découvertes faites en archéologie amazonienne, au cœur de la forêt, dans les basses terres, qui ont fait connaître l'existence de civilisations très anciennes - elles se sont développées à partir du premier millénaire avant notre ère et ont prospéré durant des siècles dont on peut difficilement affirmer qu'elles étaient l'œuvre de sociétés sans État. À tout le moins aurions-nous affaire à des cités-États ou à de grandes chefferies supposant l'existence d'une stratification et de hiérarchies sociales aussi marquées que celles qu'ont connues l'Ancien Monde et l'Afrique noire, en particulier ${ }^{15}$.

Il n'en demeure pas moins intéressant de dire quelques mots de l'argumentation à laquelle a eu recours un américaniste pour soutenir cette idée à la fois paradoxale et féconde de société contre l'État. Pierre Clastres expose ainsi quelques-uns des mécanismes permettant, selon lui, aux communautés indiennes qu'il a étudiées au Paraguay et dans d'autres pays d'Amérique du Sud, d'empêcher la constitution non pas d'un État mais d'un pouvoir autoritaire dévolu à un chef qui serait, dans la durée, en capacité d'enclencher une dynamique y conduisant. À titre d'exemple, je citerai son analyse du rôle de la guerre dans les sociétés qu'il définit comme indivises: ces sociétés du même type se livrent précisément à des batailles entre elles afin de persévérer dans leur indivision. Même si on ne doit pas oublier qu'il s'agit de pratiques belliqueuses qui n'entraînent que de faibles pertes car il faut les rapporter à la taille des populations en cause et donc au fait que les effectifs qui y prennent part sont très modestes, on voit bien que la situation politique qui en résulte ne peut qu'être des plus instables, y compris dans les communautés indiennes les plus "primitives", c'est-à-dire, en l'occurrence, celles qui seraient longtemps demeurées dans un relatif isolement à l'intérieur d'une zone géographique d'accès difficile. Quoi qu'il en soit, une preuve a contrario de cette affirmation qui implique la réciprocité entre les partenaires qui

15. Je renvoie le lecteur au remarquable numéro de L'Homme (1993, 126-128) intitulé "La remontée de l'Amazonie ». Il se reportera, notamment, à l'article de l'archéologue américaine Anna Curtenius Roosevelt: "The Rise and Fall of the Amazon Chiefdoms» (pp. 255-283). On peut y lire ceci : "Unlike today, settlements at that time seem to have been embedded within large cultural and political territories with allegiance to paramount chiefs claiming divine origin and elaborate sumptuary rights to emblems of office, certain resources and valuables, etc. » (p. 260). 


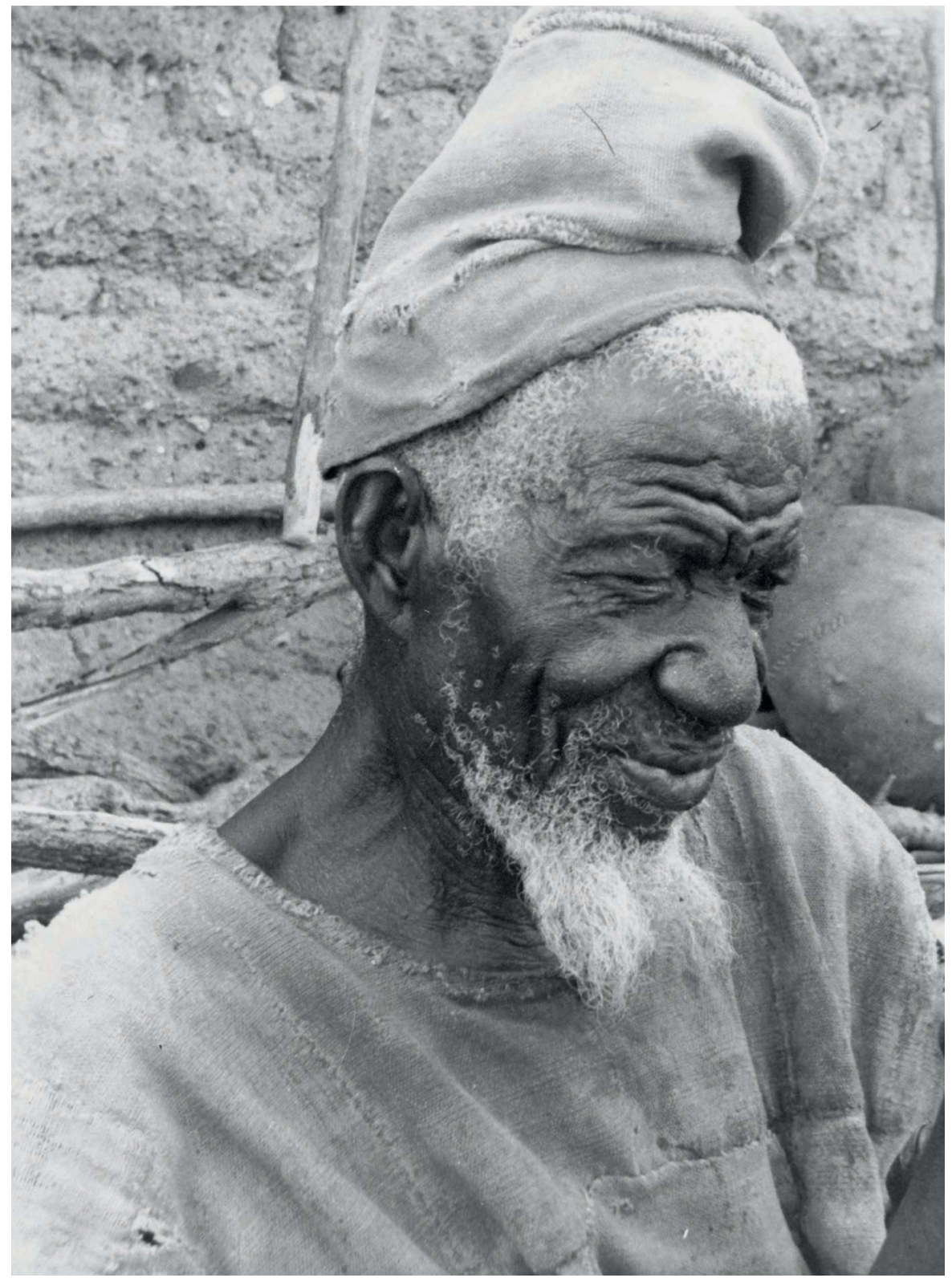



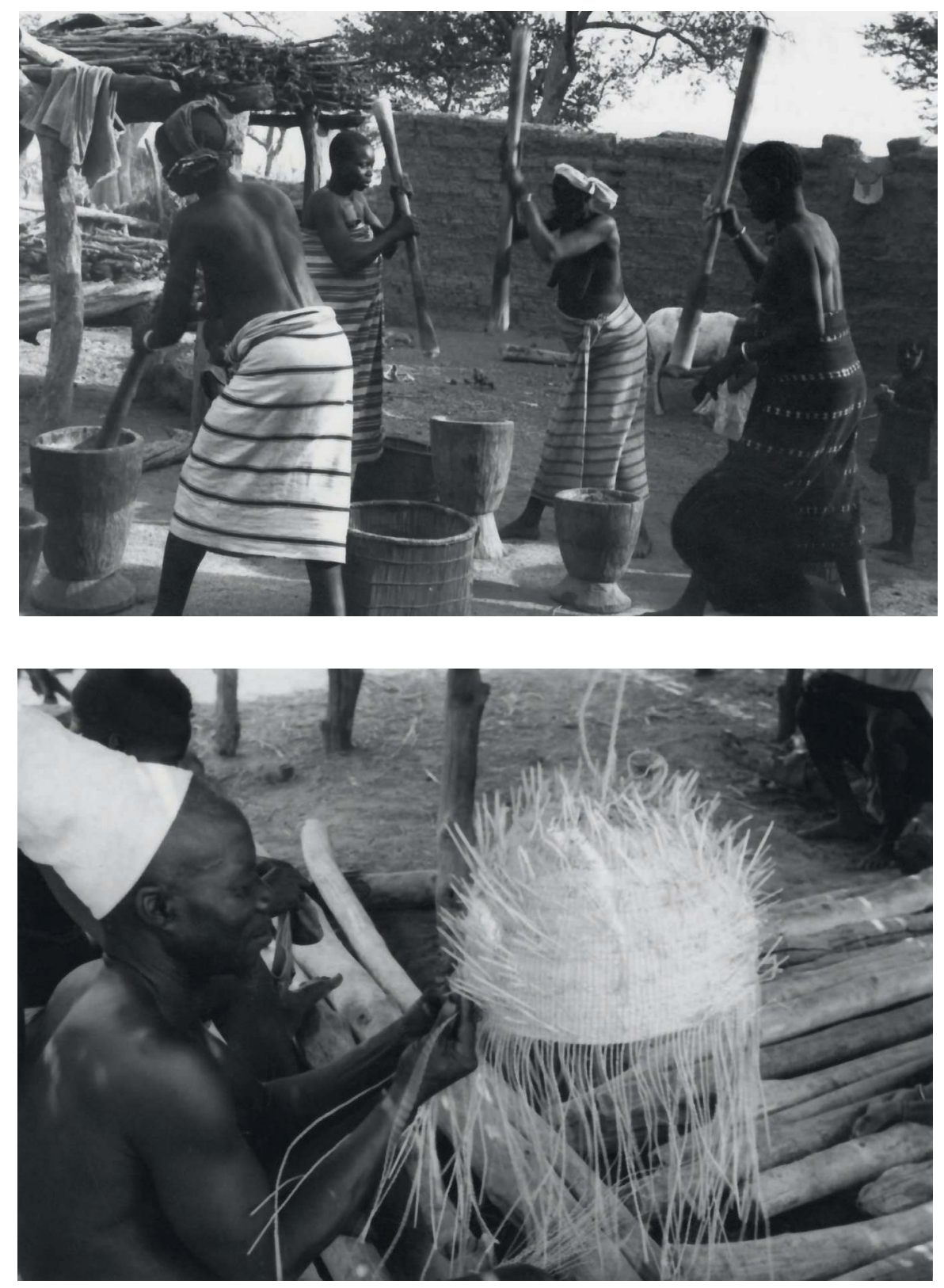
entrent en rapports guerriers est apportée par le sort qui attend l'individu ambitieux qui désire s'élever au-dessus des autres et, d'une manière ou d'une autre, s'imposer comme leur leader. Il se trouve engagé dans une poursuite sans fin d'exploits : il doit tuer de plus en plus d'ennemis pour en rapporter les scalps. Il peut alors s'enorgueillir des titres de gloire qu'il y récolte à proportion du nombre de ces précieux trophées qu'il rapporte mais sans tirer de profit politique personnel durable de ce type singulier de "capitalisation ", laquelle est toujours jugée insuffisante. Son entreprise de plus en plus téméraire le voue donc à une mort certaine et même à brève échéance tant les risques que la communauté le contraint à prendre sont grands. En m'en tenant à cette "ruse» au moyen de laquelle la dite communauté "se défend ${ }^{16}$ contre la menace que fait peser sur elle le guerrier un temps victorieux, à savoir de rendre possible l'existence en son sein d'un pouvoir séparé d'elle-même, ce qu'on pourrait désigner comme un processus de conquête du dedans mais sous la forme, dirons-nous, d'un "modèle réduit », je m’interroge sur le point de vue de Michel Izard confronté à l'analyse de situations du même ordre, c'est-à-dire faisant apparaître des contre-finalités, dans "l'espace étatique ouest-africain ".

L'idée, ou si l'on préfere, l'hypothèse d'une société "pour l'État» suppose que soient repérées dans le stade antérieur, celui d'une société de non-État, les potentialités latentes qui vont s'actualiser par la levée des défenses qui la protégeaient contre les forces qui la conduisent à accepter le cadre étatique du Moogo tel qu'il est présenté par Michel Izard. Avant d'en résumer les grandes lignes, je reviens un instant sur le problème logique qui est posé ici : peut-on dire que l'absence équivaut à un refus et peut-on donner à cet énoncé une portée théorique générale alors que, pour reprendre le langage d'Aristote, nous ne disposons pas d'une définition du concept d'État, ni en compréhension ni surtout en extension. Où commence donc l'État et où finit le non-État?

Les très précises et très fines analyses de Moogo ont bien montré, nous l'avons vu, qu'il y a continuité entre l'État et les systèmes politiques fondés sur des communautés villageoises avec ou sans chefferies (ou cheffautés, selon le néologisme de l'auteur forgé sur le modèle de royauté) plus ou moins puissantes ou sur des structures lignagères. Ces dernières étant, selon la thèse soutenue par Michel Izard et que nous avons résumée

16. Les guillemets sont là pour indiquer que la ruse comme moyen de défense n'est pas du ressort d'on ne sait quelle intentionnalité imputable au groupe qui pressentirait l'aliénation résultant de la formation de l'État, mais l'effet de croyances et de pratiques sociales qui touchent au statut du guerrier et à la signification des scalps dans ces sociétés amérindiennes. C'est en pensant à l'expression hégélienne de « ruse de l'histoire » que j'ai fait usage de ce mot. 
plus haut, à l'origine du type d'État propre aux Mossi dont l'armature est constituée par un groupe de descendance patrilinéaire (le moos buudu) en expansion constante. Expansion découlant, d'une part, des règles de dévolution du pouvoir (il faut qu'en plus du fils aîné qui succède à la mort de son père, un autre ou quelques-uns de ses autres fils soient pourvus d'un commandement local) et, d'autre part, de la segmentation des lignées qui l'accompagne avec assignation au chef qui vient d'être investi d'un nouveau territoire pris ou grignoté dans l'espace moogo. C'est ainsi que le territoire a été " conquis » et en même temps morcelé (car de nombreux " espaces interstitiels" demeurent) dans un même procès historique qui a abouti à la formation des divers royaumes mossi d'importance très inégale ${ }^{17}$. Telle est la situation politique que trouveront en face d'elles les troupes coloniales qui pénètrent au cœur de l'Afrique noire occidentale (appelée à être le futur Soudan français) à la fin du XIX siècle. Considérée d'un point de vue formel, celui des règles dont nous venons de parler, la mise en place se serait simplement faite au fil des générations et des successions au pouvoir des ayants droit, c'est-à-dire, des prétendants issus en filiation directe des rois ou chefs régnants. En réalité, il s'agit d'un processus au déroulement très lent (il aura duré de deux à trois siècles) car dépendant des facteurs multiples : ceux que nous avons mentionnés : socio-économiques, démographiques, culturels, etc., mais essentiellement de la manière dont les deux protagonistes en cause, les conquérants et les autochtones - désignés respectivement comme "gens du pouvoir» et "gens de la terre"-, se sont perçus l'un l'autre et se sont comportés l'un vis-à-vis de l'autre.

Dans un paragraphe intitulé "Problématique de la conquête ", Michel Izard indique avec une grande justesse ce à quoi le chercheur est ainsi confronté :

«Il en est de la conquête comme de la migration; il s'agit d'un phénomène dont la réalité nous échappe dans une large mesure. Lorsqu'une tradition orale nous parle de quelque chose à quoi nous donnons le nom de conquête, tout se passe comme si de part et d'autre - la tradition et la lecture que nous en donnons - il y avait accord pour constituer l'événement comme une sorte de surgissement sans cause mais dont la finalité serait si évidente qu’à elle seule elle suffirait à prédire ou à accepter comme allant de soi la réussite de l'entreprise» (2003: 136).

Après avoir noté que jamais les guerriers, aussi forts et habiles soient-ils, ne peuvent soumettre hommes et territoires sans coup férir, il ajoute:

17. Deux d'entre ces royaumes se distinguent très nettement des autres par leur taille et leur puissance politique et militaire : celui de Ouagadougou dont le souverain a été présenté comme l'« empereur des Mossi » et celui du Yatenga. 
«[...] notre information en matière de réaction armée des sociétés paysannes africaines aux entreprises étatiques - précoloniales et coloniales - nous aide à imaginer que si l'intrusion est vécue par ses acteurs comme la mise en œuvre pratique d'un rapport initial du fort au faible visant les habitants du pays à conquérir, cette certitude peut être bien vite mise à mal par une résistance s'appuyant sur une guérilla toujours déstabilisatrice pour un dispositif militaire conventionnel [...]. [S]i conquête il y a, elle revêt la forme d'un mixte d'actions de guerre et de démarches de paix. La tradition orale ne s'y trompe pas, qui mêle faits d'armes et négociations gagées sur des alliances matrimoniales nouées au meilleur niveau, en sorte que le conquérant peut se payer le luxe de présenter l'instauration politique dont il se veut l'initiateur comme relevant du souci de répondre favorablement à une demande de pouvoir émanant des futurs dominés ".

Ainsi, la conquête, événement insaisissable comme tel ${ }^{18}$, est-elle ramenée par la tradition à la conclusion d'une espèce de contrat. Un contrat qui oblige non pas des hommes soumis à obéir aux volontés de leurs maîtres et à travailler pour eux (par exemple, en payant à échéance régulière un tribut prélevé sur les récoltes ou sur le cheptel ou encore en fournissant de la main-d'œuvre pour des corvées diverses - de telles prestations ne sont cependant pas exclues) en échange de leur protection mais qui lie, selon les termes en usage chez les Mossi, les gens du pouvoir, ceux qui sont arrivés avec le naam, aux gens de la terre, gens du commun, certes, face aux nakombse, membres du clan royal, mais qui ont en charge le culte de la Terre (tenga) faute duquel le pays, en l'occurrence, le sol nourricier, irait à sa ruine. Nous avons utilisé le mot de contrat que l'on peut juger impropre en l'absence de toute référence à des concepts d'ordre juridique, mais il est utile pour indiquer qu'il est ici question d'un acte de souveraineté. Il n'est pas formulé expressément par des partenaires libres et égaux, mais il est doté d'une réalité substantielle résultant de leur indissoluble union ${ }^{19}$. Néanmoins, il s'agit bien d'une souveraineté du pouvoir, d'une

18. Cette proposition s'applique de façon générale à l'histoire de l'Afrique précoloniale. Le cas des guerres saintes menées au XIXe siècle au nom de l'islam fait exception et, notamment, le jihad proclamé par Ousman dan Fodio dans les toutes premières années du siècle. Je renvoie à l'analyse que Michael G. Smith (1970), dans son ouvrage Government in Zazzau, a consacrée aux transformations structurelles du système politique de cette cité hawsa (Nord-Ouest du Nigeria) consécutives à la conquête peule.

19. À propos de l'idée de complémentarité fonctionnelle à quoi peut nous faire penser cette union, Michel Izard observe qu'en l'occurrence, «elle ne rend compte que de la meilleure part de ce qui demeure un affrontement, que celui-ci puise ses origines dans l'histoire ou s'éclaire par des considérations d'ordre économique et social. Gens du pouvoir et gens de la terre vivent dans des univers de pensées, d'actes, de paroles qui sont irréductibles l'un à l'autre, de sorte que le couplage divin renvoie moins à un équilibre harmonieux qu’à un déséquilibre perpétuellement en quête d'harmonie " (2003 : 121). Il faut aussi citer ce passage de L'Odyssée du pouvoir, où l'auteur écrivait ceci : «Le pacte qui associe gens du pouvoir et gens de la terre peut être scellé dans la complémentarité vraie [c'est moi qui souligne], double discours proféré à l'unisson, fondant bien davantage une association entre deux groupes que la domination de l'un des deux par l'autre» (1992: 42). 
souveraineté d'État qui maintient la différence dans cette union du ciel et de la terre et en proclame la légitimité qu'elle fonde sur l'idée de l'Un, le roi intronisé, descendant en ligne directe du héros fondateur et détenteur suprême du naam, lequel en lui-même est à la fois une représentation et une incarnation de cette représentation dans la personne du roi du pouvoir, du naam absolu, si l'on peut dire, celui qui appartient à l'essence de Wende. Ce nom est celui du dieu céleste, identifié, présentifié par le soleil. Naaaba Wende (le roi ciel ou roi soleil) fait couple avec Tenga, la Terre (déesse appelée Napaaga Tenga, Terre, femme de chef). Les deux divinités sont invoquées, et le plus souvent Wende, mais Wende est beaucoup trop loin des hommes pour que leurs sacrifices ou leurs offrandes puissent l'atteindre et influer sur le cours des choses, seule Napaaga Tenga a droit à des "prêtres, autels et rituels, un culte en un mot» (2003: 121). Les rois mossi et tout particulièrement les deux plus importants que sont le Moogo naaba (celui, je le rappelle, qu'on avait gratifié du titre d'empereur des Mossi) et le roi du Yatenga, ont indéniablement un caractère solaire (qu'ils partagent avec de nombreux autres souverains d'Afrique noire), ce qui a conduit maint chercheur à s'interroger sur un lien éventuel avec les conceptions égyptiennes touchant à la figure cosmique du Pharaon. Vieux débat parmi les africanistes dont on ne saurait dire qu'il est clos et je crois nécessaire de citer à ce propos la note ajoutée par Michel Izard:

«Il convient dans ce domaine de ne pas forcer l'interprétation des faits relatifs à une théâtralité royale dont les éléments "solaires" sont trop disparates et discrets pour être intégrés en un système de représentation cohérent. Au mieux peut-on noter que l'axe est-ouest qui figure le tracé de la destinée humaine peut avoir valeur de projection sur le sol de la courbe décrite dans le ciel par le soleil de l'aube à la fin du jour, la position du soleil à son zénith correspondant sur le sol à la position du roi, qui fait "centre" dans le royaume" (ibid. : 120).

Prudence compréhensible tant est forte la tentation, si l'on s'attache à interpréter le symbolisme royal, de passer d'une similitude de traits entre l'Afrique noire et l'Égypte antique à l'affirmation d'une filiation historique seulement probable mais strictement indémontrable. Indémontrable jusqu'à ce que de nouvelles découvertes nous disent comment ce legs égyptien a pu se perpétuer à travers les millénaires et traverser l'immensité du désert et de la zone sahélo-soudanienne. Pour nous, le problème n'est pas là. Ce qui nous importe est que Michel Izard ait mis en évidence l'articulation des deux ordres, celui qu'il désigne comme l'ordre étatique et qui appartient au ciel où règne le soleil et celui des gens de la terre sur laquelle, changeante en fonction des différentes phases de ses apparitions nocturnes tout au long du cycle mensuel, la lune répand sa clarté. La lune 
dont dépend, mais pas entièrement, la mesure du temps auquel s'accordent les saisons et le cycle annuel des travaux agricoles qu'accompagnent les rites royaux faute desquels ils se révéleraient, cela ne fait aucun doute aux yeux des paysans, totalement improductifs. C'est donc au calendrier, miroir de la cosmologie et code de l'organisation commune du travail où l'espace et le temps se rejoignent pour ne faire qu'un, que nous allons nous intéresser maintenant.

Le rappel de quelques données astronomiques ${ }^{20}$ est indispensable. Le mot moré kilika correspond à la nouvelle lune, laquelle suit d'au moins vingt-quatre heures la nouvelle lune astronomique. Une année solaire comprend douze apparitions de la nouvelle lune mais l'année lunaire retarde de 10,87 jours (l'épacte). Il y a coïncidence approximative entre la durée de trois années solaires et celle de trois années lunaires augmentée d'une lunaison, soit une durée de trente-sept mois lunaires. Pour maintenir les mois lunaires dans une position relativement stable par rapport à l'année solaire, il faut intercaler un mois lunaire supplémentaire après trois années révolues, la troisième année comprenant donc un treizième mois. Dans le calendrier du Yatenga, ce mois intercalaire (embolisme) est introduit toutes les trois années solaires par doublement de la septième lunaison, laquelle est appelée kizalogo, "lune vide». Ce doublement intervient en début de saison des cultures (mai-juin). L'année solaire commence avec l'apparition de la nouvelle lune la plus proche du solstice d'hiver (en 1964, cela tombait le 6 décembre): c'est le mois de filiga, c'est-à-dire du "remerciement " adressé à la terre, aux ancêtres. La fête est marquée par les salutations faites au roi par des groupes détenteurs d'une parcelle plus ou moins importante du nam (terme qui désigne le pouvoir royal légitime et tout pouvoir qui en procède) : les serviteurs royaux, les maîtres de la guerre, et les membres de l'aristocratie royale, nakombse, les fils de lignée royale. Les cinq lunes suivantes portent les noms de cinq localités du royaume et donnent lieu à une série de rites de fertilité, le cycle de bega. C'est le roi qui, à l'approche de chaque nouvelle lune du bega, ouvre la phase correspondante du cycle cérémoniel en faisant remettre au maître de la terre du village éponyme du mois du sel et des graines de néré. Il fournit ainsi les condiments de la sauce et il incombera aux "gens de la terre " d'obtenir le mil dont ils donneront au roi les prémices. 
Après les lunes du bega, nous avons une ou deux lunaisons appelées kizalogo qui nous mènent à l'articulation des lunes cérémonielles de saison sèche et de celles de la saison des pluies durant laquelle se font les gros travaux de culture. Les trois mois qui viennent sont désignés par les activités suivantes: semailles, premier et deuxième sarclage ou binage. Le quatrième mois (la onzième lune, celle d'octobre) est appelé yaalum, le mil, mais en fait il s'agit du sorgho blanc qui arrive alors à maturité. Survient alors le moment de la fête royale de koom filiga, le filiga de l'eau, entendons la bière de mil ; le roi peut maintenant consommer le grain de la nouvelle récolte. L'année s'achève avec le mois de tiido, mot qui désigne à la fois le deuxième des trois grands regalia du Yatenga et l'autel des buguba, les prêtres maîtres de la pluie et gardiens de l'esprit du mil qui, à l'apparition de cette nouvelle lune, célèbrent leur rituel après quoi est levé l'interdit touchant à la consommation de l'eau des puits qui pesait sur eux depuis la première précipitation de la saison d'hivernage.

\section{Espace et calendrier}

Une correspondance est établie entre le tracé géographique du circuit des cinq villages du cycle du bega suivi dans le sens chronologique $\mathrm{du}$ calendrier et les circumambulations rituelles $\mathrm{du}$ roi autour du palais (nayiri, "maison du roi»), à l'occasion des rites de "remerciement»: donc, dans le sens est-ouest. Départ de la porte de l'est qui est celle des captifs et des femmes et arrivée par la porte de l'ouest qui est celle des Moose (l'ensemble des descendants d'ancêtres appartenant au lignage fondateur du royaume), les dignitaires et les serviteurs du palais. Notons que le sens de la circumambulation est inversé à l'occasion des funérailles. Le sens est-ouest est aussi celui dans lequel le roi se déplace de village en village au cours de son voyage d'intronisation, le ringu (le "royaume»). "Ces parcours circulaires", nous dit Michel Izard, "imposent l'image d'un royaume assimilable à un cercle dont le centre est le lieu où se tient le roi ». Symbolisme politique et symbolisme cosmique sont ici condensés : l'espace du royaume est tracé au moyen d'un rituel qui inscrit sur le sol une forme circulaire. Cette circularité est inséparable de l'orientation de l'axe est-ouest dont le sens est celui de la vie alors que l'axe de sens opposé est celui de la mort, on vient de le voir. Bien sûr, cette circulation est-ouest qui est le sens du mouvement apparent du soleil dans le ciel, implique une homologie entre les rapports roi-terre et soleil-ciel et, dans le même mouvement, l'identification entre le soleil dont on peut dire métaphoriquement qu'il règne au ciel, avec Naba Wende. Naba Wende, c'est-à-dire Dieu, le Dieu céleste qui, nous l'avons vu, s'oppose à la puissance tenga, 
la Terre. L'auteur note qu'il ne faut pas oublier qu'on a ici affaire à une transformation métaphorique d'une métonymie touchant aux rapports spatiaux entre le cercle terrestre et la sphère céleste. Avec le cycle du bega qui concerne les cinq principales maîtrises de la terre qui se partagent l'espace entier du royaume, on voit que le roi, dont l'une des fonctions les plus spécifiques est de donner l'impulsion initiale à chacune des phases de ce grand rituel de fertilité, est à «l'articulation des mondes du pouvoir et de la terre». Mais il faut rappeler que sa position centrale comme homologue à celle du soleil s'oppose à la centralité qui est la sienne par rapport à ces cinq maîtres de la terre. Les rites de fertilité vont du centre vers la périphérie et le mouvement s'inverse au moment des salutations annuelles au roi car alors le mil obtenu grâce à l'accomplissement de ces rites est transporté des villages vers la résidence royale. L'argumentation de Michel Izard est la suivante : "le roi est sur l'espacesol comme le soleil zénithal est dans le ciel diurne; il est le "centre" d'un cercle que l'on peut définir comme la projection du ciel diurne sur la terre». Autrement dit, la position du roi est celle d'un point par lequel passe l'axe est-ouest (c'est la métonymie qui donne lieu à la métaphore roi-soleil). Évidemment, la lune n'occupe pas dans le ciel nocturne une position analogue à celle du soleil dans le ciel diurne, elle ne définit aucune centralité sur l'espace-sol ni aucun axe. "Par contre ", nous dit l'auteur, "c'est la projection du mouvement de la lune sur l'espace-sol qui en définit le pourtour circulaire au long des cinq mois consécutifs». Ce sont les villages éponymes des nouvelles lunes correspondantes qui décrivent sur le territoire du royaume un cercle dont le roi est le centre. Ainsi «le cycle du bega nous montre que la lune est liée à la sacralité de la terre, tandis qu'au soleil est associée l'idéologie du pouvoir. Le pouvoir et la terre ont respectivement leur spatialité et leur temporalité propres». Michel Izard conclut son étude consacrée au calendrier soli-lunaire et à la manière dont les Mossi du Yatenga ont résolu les problèmes qu'il soulève, en affirmant - mais le saut qu'il fait ici dans son argumentation peut sembler arbitraire - que la temporalité du pouvoir est celle de l'histoire, il s'agit donc d'un temps cumulatif, quant à celle de la terre, cyclique par excellence, elle est répétitive et sans fin. La proposition suivante: "Le calendrier du Yatenga rend compte de l'articulation nécessaire entre deux temporalités, qui est l'articulation entre deux spatialités; le temps n'est pas pensable, mesurable, hors de l'espace" indique bien, me semble-t-il, qu'en invoquant le temps historique, le temps de l'événement, il saute un chaînon logique. Le caractère solaire du roi est certes une symbolisation du pouvoir, mais je ne crois pas qu'il faille l'interpréter comme relevant d'un discours idéologique qui 
émanerait $d u$ pouvoir ${ }^{21}$ en tant qu'il s'inscrit dans un devenir historique déterminé. Le processus de conquête (qui, en l'occurrence, ne se réduit pas à un phénomène de progression purement guerrière sur le terrain, nous y avons longuement insisté) et de domination d'un espace demeuré sous la maîtrise des "gens de la terre", arrivé à son terme, engendre un espace-temps politique inséparable de l'espace-temps cosmique mais non identique à celui qui s'est révélé dans l'étude du calendrier.

Pour prendre la mesure de l'importance de la notion d'espace-temps cosmique comme fondement du calendrier rituel aussi bien chez les Moose que dans de nombreuses sociétés d'Afrique occidentale, je me référerai au cas des Évhés du Togo qui ont fait à cet égard l'objet d'une étude approfondie que l'on doit à Albert de Surgy (1990) et qui mérite d'être rapportée avec une certaine précision.

Les Évhés sont l'une des populations appartenant à la grande aire culturelle du Bénin comprenant, entre autres, les Yoruba, les Fon et les Edo qui furent les créateurs de puissants royaumes dont certains dominèrent cette région du golfe de Guinée jusqu'à la fin du XIX siècle. La royauté occupait donc la place centrale dans les institutions politiques de ces sociétés qui, tournées vers la guerre et le commerce continental et maritime (celui des armes et des esclaves, notamment, mais aussi des noix de cola), devaient disposer d'un pouvoir fort. Cependant, ce n'était pas le cas des Évhés chez qui le pouvoir, à proprement parler, était exercé par un conseil des aînés de lignage. Ce même conseil désignait, à la suite de longues consultations divinatoires, une sorte de grand chef, fiagâ, que Surgy propose d'appeler un "prêtre-roi » car il représentait et, semble-t-il, représente toujours, la plus haute instance religieuse qui, du cœur de sa forêt (un lieu-dit Bè, du nom du village qui est aujourd'hui un quartier de la ville de Lomé) où il est reclus, rayonne sur un vaste territoire dépassant les frontières strictement tribales. Quel est ce personnage qui, en définitive et malgré les apparences, n'est pas si différent des souverains qui furent à la tête des royaumes guerriers que nous venons de mentionner ? Nous pouvons nous faire une idée précise de son rôle en reprenant les sous-titres que Surgy donne à chacun des paragraphes qui composent son article :

21. Michel Izard insiste beaucoup sur la différence entre le concept de nam, le pouvoir légitime lié à la conception de la sacralité de celui qui le détient en tant que souverain, et panga (terme d'origine mande que l'on retrouve dans faama, le chef, le roi) la force brutale qui, comme attribut du pouvoir royal, n'en est pas moins pour autant légitime et nécessaire, puisque sans elle le pouvoir n'est rien. Je renvoie donc le lecteur aux ouvrages de l'auteur et, notamment à Moogo. L'émergence d'un espace étatique onest-africain au XVI siècle (2003) que je considère comme une contribution nouvelle et fondamentale - à la fois anthropologique et historique - à la problématique de l'émergence et du déploiement de l'État en Afrique noire. 
1) "Le prêtre-roi des Bè ». Cette désignation est justifiée car il est, en effet, le prêtre du dieu Nyigblé ; cette divinité dite "forgeron de dieu " a en charge la pluie et la fécondité et elle est considérée comme féminine. Le côté féminin de notre personnage est souligné par le fait que dans sa forêt, il n'est entouré que de femmes: durant son règne, il y résidera avec deux promotions successives d'une trentaine d' "épouses", fiasi, des adolescentes qui sont là pour célébrer le culte de Nyigblé en apprenant des chants et des danses qu'elles exécuteront en son honneur. Elles sont les prêtresses de ce dieu et traversent ainsi une période d'initiation comme le feraient les adeptes de tel ou tel vodu.

2) «Une royauté purement symbolique». Le prêtre-roi est éloigné des affaires de toutes sortes dont s'occupent les autorités politiques, c'està-dire, en l'occurrence, le conseil des anciens. Il n'empêche qu'il le préside, à l'occasion, pour que celui-ci bénéficie " de l'énergie spirituelle qui émane de sa personne». Et Albert de Surgy ajoute une remarque essentielle: "Nous constatons effectivement que la durée de règne d'un prêtre-roi est en général précédée et suivie d'un interrègne plus long ». Nul n'en a cure car cela n'a pas d'incidence effective sur le cours des choses. Il est reclus, il n'a pas le droit de travailler, les rapports sexuels lui sont interdits, il ne s'adresse aux hommes qui viennent le consulter que par le truchement d'un porte-parole.

3) "Un roi plus efficace mort que vivant». C'est dans ce paragraphe qu'apparaissent des considérations précises sur le comput du temps. Albert de Surgy indique d'abord qu'il est dit (par des informateurs prudents qui exigent de lui la plus grande discrétion) que le régicide rituel était pratiqué au plus tard au cours de la septième année de règne et il ajoute que ses enquêtes sur la durée de règne du dernier roi-prêtre confirment qu'il en était bien ainsi. En prenant pour unité de compte non pas l'année mais la lunaison, il établit la chronologie suivante: la consécration du nouveau prêtre-roi a lieu au moment où la constellation des Pléiades fait son apparition à l'ouest, dans « la nuit noire qui sépare la onzième et la douzième lune ». Il est alors conduit dans une première forêt sacrée où il séjournera pendant trente-trois lunaisons. Après cette période d'initiation, il se rend dans la forêt de Bè où il est pleinement investi de sa charge, mais le conseil des anciens attend six lunaisons pour proclamer l'événement. C'est alors que commence une autre période de quarantequatre lunaisons au terme de laquelle il devra mourir, ce qui fait que la durée totale de son règne aura été non pas de sept ans exactement mais de soixante-dix-sept lunaisons. À cette durée correspond une première promotion d' $d$ épouses rituelles" qui l'aura accompagné pendant quarante-quatre lunaisons, puis une deuxième qui l'aura fait pendant 
trente-trois lunaisons + onze, représentant, elles, le temps nécessaire pour clore les funérailles. Le parallèle existant entre les quarante-quatre lunaisons d'initiation de chacune des deux promotions d'épouses et la période d'initiation d'une adepte à son vodu s'applique également au prêtre-roi car on peut dire que comme pour les fiasi, il s'agit pour lui d'une initiation qui dure aussi longtemps que celle de l'adepte d'Afa, c'est-à-dire du devingéomancien ${ }^{22}$. Qu'on me permette de citer assez longuement notre auteur :

"Cela signifie que la date à laquelle il est bon que le prêtre-roi meure est analogue à la fin d'une période de formation ayant fait suite à un cycle de simple initiation. Tout se passe comme s'il était soigneusement formé pour n'exercer qu'une fois mort le rôle que l'on attend de lui, celui d'un irremplaçable intermédiaire avec l'au-delà, élevé au rang de divinité. Si les interrègnes sont si longs, c'est qu'il est jugé plus efficace mort que vivant, mais ne le demeure que durant un certain cycle temporel à l'issue duquel son successeur doit à son tour être sacrifié pour le remplacer» (de Surgy 1990 : 105).

4) «L'intronisation du prêtre-roi et le calendrier». Sans entrer dans trop de détails et en reconnaissant que je suis plutôt en peine de juger, s'agissant de comprendre le calendrier des Évhés, du bien-fondé de la référence au cycle de Méton $^{23}$, je résumerai très brièvement les données

22. À ce propos, il est intéressant de faire un rapprochement avec l'un des systèmes de classes d'âge que l'on trouve chez les Yoruba du Nigéria, celui qui a été étudié par Andrew Apter (1992) dans la ville d'Ayede. Il met en évidence un modèle dans lequel les 16 classes générationnelles portant chacune un nom et ayant une devise correspondant à un événement marquant de l'histoire de la cité-État. Elles ont toutes une durée de seize ans et l'on obtient ainsi un cycle complet égal à $16 \times 16=256$. Ce chiffre est à mettre en rapport avec la numérologie ésotérique qui caractérise le système divinatoire d'Ifa. Celui-ci fonctionne avec 16 odu (versets ayant valeur oraculaire pour le client), 16 noix de palme dont le jet détermine le message d'Ifa. Les 16 figures multipliées par les 16 maisons (les valeurs sémantiques retenues par le système géomantique) donnent les 256 combinaisons possibles. En outre, ce chiffre 16 est aussi associé au mythe des 16 royaumes primitifs des Yoruba, fondés par les 16 fils d'Oduduwa, le premier roi d'Ifé. Andrew Apter ne fait aucune allusion à des considérations astronomiques que les Yoruba rattacheraient à leur système divinatoire. Ce qu'il faut retenir de ces faits, c'est l'existence d'un symbolisme numérologique appliqué à l'organisation du temps social qui comporte de façon implicite une articulation des classes d'âge avec la royauté. S'il n'y a pas de régicide rituel, il y a en permanence menace d'assassinat politique dont la décision est entre les mains de cette espèce d'État-major qui est à la tête du système des classes dont la fonction militaire était tout à fait essentielle. Je renvoie aussi le lecteur à un passage de l'ouvrage de Bernard Maupoil (1988: 216-227) : «Calendrier du roi et du peuple: Hwezầ ". Sur les faces d'une longue planche rectangulaire sont pyrogravés les $16 d u$ capitaux, c'est-à-dire les 16 signes géomantiques.

23. Méton est un astronome athénien du V viècle avant notre ère. Il découvrit (en tout cas, cette découverte porte son nom) qu'il existe une correspondance presque exacte entre un nombre donné d'années solaires (19) et un nombre de lunaisons (235). Si 19 années solaires $=235$ lunaisons, il en résulte que tous les 19 ans, la lune doit se trouver exactement (à peu de chose près) au même endroit dans le ciel. Il s'agit là d'une véritable performance de la part d'un observateur qui ne disposait d'aucun autre moyen que ses yeux pour contempler le ciel. Il semble que la connaissance de ce cycle ait répondu aux besoins des Grecs pour fixer la date des Jeux olympiques. L'Église s'y référa pour fixer son calendrier des fêtes qui est un calendrier lunaire comme celui qui est en usage dans la religion juive. 
fournies par de Surgy. L'année évhé commence avec la cinquième lune qui suit l'apparition des Pléiades à l'Est. Comme il peut parfois s'avérer difficile de déterminer cette date, il faut une autorité pour la fixer et ce sont les prêtres de Nyigblé, la divinité, on l'a vu, liée à la forge, qui en sont investis. Du choix qu'ils font découle tout le calendrier rituel valable pour tous les Evhés. Il s'ensuit aussi, observe de Surgy, que les prêtres sont inévitablement confrontés à la nécessité astronomique suivante :

" [...] du fait que 19 années solaires comportent exactement (à peu de chose près) 235 lunaisons $-12 \times 19=228+7-$ (cycle de Méton), la minimisation du décalage entre des années luni-solaires comportant un nombre entier de lunaisons et des années solaires (celles du cycle agricole) est assurée en insérant 7 années de 13 lunes parmi 12 autres années de 12 lunes» (Ibid.).

Or, malgré les à-peu-près de sa chronologie pour déterminer les dates auxquelles, depuis l'origine (c'est-à-dire l'implantation de la «tribu» des Bè sur le littoral atlantique), se seraient succédé les prêtres-rois, Albert de Surgy pense avoir établi qu'un intervalle de 19 ans séparant deux intronisations successives devait être la norme. À partir de là, il s'estime en mesure de "risquer ", nous dit-il, l'hypothèse que les prêtres avaient perçu un lien entre la nécessité d'introniser un nouveau prêtre-roi et le problème que leur posait l'ajustement du calendrier. Et il enchaîne sur une hypothèse, à mon sens, beaucoup plus risquée encore et qu'on serait tenté d'appeler l'hypothèse d'une variante africaine du sacrifice aztèque :

«Chaque fois que l'apparition d'une nouvelle lune pourrait être déclarée antérieure ou postérieure au lever héliaque des Pléiades, qui sont assimilées à la matrice du monde, le moment paraîtrait venu de consacrer un nouveau prêtre-roi dont l'existence ou le sacrifice serait de nature à redonner une impulsion au cycle du temps pour éviter qu'au lieu de se déployer il ne réintègre son origine» (Ibid. : 107).

\section{Un roi prisonnier du temps}

La nouveauté de cet éclairage sur le régicide rituel tient paradoxalement à ceci qu'un acte par lequel une société fait un choix radical dans la fixation de son calendrier - je tiens à souligner que le mot calendrier est ici à prendre dans son sens étymologique romain, c'est-à-dire celui d'appel à tous les membres de la cité pour que les rites soient observés en temps voulu - est analysé en fonction précisément des questions touchant à la nature du calendrier lui-même. Un même temps cyclique réglant les travaux et les jours règle également la durée des règnes qui dépend ainsi des rythmes cosmiques et qui révèle la nature profonde de la royauté et, d'une manière générale, du pouvoir, que ce soit en Afrique ou ailleurs. 
Le fait que dans la littérature ethnographique consacrée à l'Afrique noire, l'exemple des Évhés s'avère, jusqu'à nouvel ordre, plutôt exceptionnel, n'enlève rien à l'intérêt général qu'il présente et conduirait plutôt à se demander pourquoi les ethnologues avertis de l'importance de cette question de l'articulation du comput du temps avec le système du pouvoir comme fonction symbolique et comme action dans le réel, n'ont pas fait plus d'efforts pour recueillir des données du même type que celles d'Albert de Surgy.

Néanmoins, les chercheurs n'ont généralement pas manqué de remarquer que les sociétés paysannes africaines avaient résolu d'une façon ou d'une autre les problèmes d'ajustement que soulèvent les calendriers luni-solaires qu'ont adoptés la plupart ${ }^{24}$ d'entre elles. Dans un autre numéro de la revue Systèmes de pensée en Afrique noire consacré aux "Calendriers d'Afrique» (1986), Michel Izard en rappelait les données astronomiques. Car, s'il est vrai qu'on n'y trouve rien de comparable aux pratiques des Jukun, des Chamba ou des Moundang, on peut y déceler une forme "éclatée ", dirais-je, de régicide dans la mise à mort d'une victime sacrificielle substitutive, le cheval du roi, tulubere weefo, et l'élimination du fils aîné du roi défunt dans le rituel du kurita qui équivaut à une mort d'une sorte de doublure du roi.

J'en reviens aux rapports du calendrier évhé avec le temps de règne assigné au prêtre-roi de Bè. Je résume le dernier paragraphe de son texte intitulé : "Centralité et effacement de la personne royale». Albert de Surgy s'efforce de montrer que la conception du calendrier est inséparable de la structure de la société, ou plutôt d'un modèle idéal que celle-ci projette dans l'espace où elle dessine une figure homologue à celle qui permet de représenter le temps astronomique. Lignages et familles forment un ensemble clanique ordonné : les différents lignages comprenant quatre sous-groupes (des familles étendues), sont attachés à une même portion du territoire; ils se répartissent en douze clans, lesquels se subdivisent en quatre sous-ensembles de trois clans. Ces sous-ensembles constituent des unités quasi autonomes de partenaires dans les échanges matrimoniaux.

24. Une exception, qui n'a malheureusement pas fait l'objet d'études approfondies, nous est fournie par les Ashanti du Ghana qui ont combiné la semaine de six jours avec celle de sept jours pour donner un «mois » de quarante-deux jours, divisions du temps qui ne sont pas sans évoquer, malgré les différences importantes qui les séparent, celles qui avaient cours chez les Aztèques. J'indique au lecteur que je rédige par ailleurs un travail comparatif sur la fête des prémices dans deux sociétés (les Swazi et les Ashanti) qui comporte des réflexions sur le comput du temps chez les Ashanti. Les Swazi ont adopté l'année luni-solaire. Leur rituel royal des prémices implique que les épreuves imposées au roi apportent une aide, une énergie renouvelée au mouvement du soleil qui semble donner des signes de faiblesse au moment du solstice d'hiver, moment où il est censé "se retirer dans sa hutte". 
C'est ainsi que s'explique que la "tribu " bè soit dirigée par un collège de douze prêtres et prêtresses au nombre desquels il faut compter le grandprêtre, le prêtre-roi.

Parmi les douze subdivisions de l'unité politique idéale, celle à laquelle se rattache le prêtre-roi occupe une position centrale mais retranchée, analogue à l'espèce de retranchement de la partie d'une période de 12 x 3 = 36 années lunaires dont n'est visible que le cycle de 33 années lunaires à l'issue duquel le début de l'année lunaire a presque rétrogradé d'un tour complet sur le cycle de l'année solaire et où il semble avoir besoin de reprendre vigueur.

Pourquoi les prêtres de Nyigblé ressentent-ils ce besoin de "venir en aide" aux corps célestes afin qu'ils ajustent leurs cycles divergents? De façon très significative, de Surgy répond en commençant par ces mots :

"Il suffirait que des spécialistes du calendrier aient élevé au rang de paradigme cette quasi-équivalence de 33 années lunaires à 32 années solaires, ou années réelles correspondant au cycle des travaux agricoles, pour qu'ils en soient venus à estimer que la perpétuation de tout cycle fondé sur la distinction de 12 catégories ne pouvait être que par une impulsion à son origine depuis l'une de ses catégories escamotée à cet effet par retrait au centre, c'est-à-dire dans cette partie retranchée ou invaginée du cycle calendaire dont il vient d'être question ».

Muni de ces données qui, pour une bonne intelligibilité, demanderaient des développements beaucoup plus précis, on peut revenir au personnage du prêtre-roi. On a vu qu'il incarne un principe divin, le forgeron mythique Nyigblé dont nous savons qu'il a en charge d'harmoniser "les activités des deux grands distributeurs des forces vitales dans le monde que représentent la lune et le soleil». À ce titre, "il garantit la pérennité des cycles temporels et le renouvellement des générations, assure l'articulation et l'assemblage des éléments de la mécanique cosmique ou sociale et, de ce fait, se situe en dehors d'elle, y demeurant totalement irrepérable ». Si cette interprétation de la mise à mort rituelle à laquelle ce prêtre-roi est voué n'est pas si éloignée, en définitive, de celle de James G. Frazer (entendons : l'existence d'un lien entre une personne séparée, retranchée du reste de la société et qui est ainsi dotée de la capacité, par le sacrifice, de soutenir un ordre du monde, un ordre de la nature conforme aux besoins vitaux de l'homme), elle s'en distingue en ceci qu'elle donne un contenu intellectuel véritable à cette idée d'ordre de la nature, contenu intellectuel que lui donnent les Évhés eux-mêmes par l'intermédiaire de leurs devins, de leurs sages. Car cette idée ne peut avoir d'efficace, déterminer des attitudes et des comportements que dans la mesure où elle est présente et agissante dans la culture évhé, comme dans bien d'autres, en Afrique et ailleurs. 
Cela étant, il ne s'agit nullement de négliger l'aspect dialectique de l'analyse d'Albert de Surgy. Le prêtre-roi de Be est marqué, on l'a vu, par son retrait dans l'espace - une portion de forêt inculte et comme dotée d'un statut d'exterritorialité -, par sa passivité, son désengagement des affaires du monde et par sa transformation en "mauvais mort " après son décès. Le mauvais mort est enterré de nuit sous le couvert des arbres alors que les autres le sont de jour et en pleine savane. Il n'exerce ni une activité d'ancêtre que l'on peut implorer ni celle d'un fantôme errant et faiseur de trouble:

"L'exceptionnelle puissance qui lui est attribuée est en fait proportionnelle à la sorte d'annulation de lui-même à laquelle il doit consentir. C'est en effet en vertu de son abstention de toute activité engagée qu'il lui est possible de s'identifier à la grande Cause $[s i c]$ agissant dans le monde, retranchée de ses effets, et de se poser ainsi en libre Agent [sic] par rapport à ce qu'il advient non seulement sur terre mais aussi au pays ancêtres et dans l'étendue intermédiaire entre les deux » (de Surgy 1990 : 107).

Il n'est apparemment question ici ni d'État ni de pouvoir d'État, l'ordre proprement politique étant, dans l'analyse de de Surgy, subordonné à l'ordre de la puissance au sein duquel elle est englobée. La puissance, elle, ressortit au plan cosmique, celui des corps célestes dont le mouvement régit toute vie terrestre. L'abstention ou, si l'on préfere, le désengagement du prêtre-roi qui renvoie, en l'occurrence, aux problèmes d'ajustement des cycles lunaire et solaire, est à penser comme des équivalents de l'interrègne (dont la durée excède celles des règnes), et du même coup, de l'identification du roi à un mort ${ }^{25}$ et, enfin, du régicide rituel. Ce sont autant de variantes d'une idée de puissance capable d'agir comme Cause en s'inscrivant dans le cours de la nature, pour reprendre le concept utilisé par notre auteur. Est-ce à dire que ces modalités de la puissance excluent le pouvoir de l'État dans l'exercice de ses fonctions de gestion des affaires communes comme dans celles de coercition?

Entre la place faite par Michel Izard aux implications politiques du calendrier chez les Mossi du Yatenga et l'analyse d'Albert de Surgy consacrée au thème du calendrier chez les Évhés du Sud-Togo, nous pouvons relever une différence qui nous ramène à la question de la nature du pouvoir politique dans ce type de royauté. Selon de Surgy, les Évhés avaient à résoudre le problème de l'ajustement de leur calendrier dont, pour dire les choses très rapidement, la mise à mort rituelle du prêtre-roi constituait la solution. Or, chez eux, on ne peut pas parler d'opposition

25. Albert de Surgy, de manière tout à fait pertinente, renvoie dans une note à une analyse de Michel Cartry (1987) dans son grand texte sur "Le suaire du chef ». Nous l'avons nous-même commenté dans le numéro 6 de la revue Incidence (2010). 
entre la centralité du personnage royal, homologue de la centralité solaire, et la situation sur la périphérie des maîtres de la terre dont les rites sont liés au cycle lunaire. On s'en souvient, la centralité du prêtre-roi, c'est son effacement même (figuré par la partie invaginée du cercle figurant le cycle calendaire) et, quant au pouvoir politique, il est exercé par les conseils de dignitaires (les Anciens) qui représentent les groupes claniques et lignagers qui composent la «tribu " des Bè. Mais la manière dont les rapports entre ces groupes se trouvent projetés sur le territoire où ils dessinent une image circulaire homologue d'une représentation du calendrier rituel est à rapprocher de la figure que dessine la ligne qui relie les cinq villages du cycle du bega. L'existence d'un pouvoir royal franchement politique et détenu dans sa plénitude par le souverain régnant auquel fait face, mais dans une relation de complémentarité, l'autorité strictement rituelle des maîtres de la terre, ne suffit pas cependant à faire disparaître, chez les Mossi, la nécessité de conserver une forme symbolique de régicide rituel ${ }^{26}$. Est-ce à dire que seuls les chefs dépourvus de pouvoir politique effectif sont mis à mort rituellement? La réponse est évidemment négative et si cette question se repose sans cesse, c'est qu'il est difficile d'échapper à la tendance à la considérer en termes d'évolution sociale, voire de "progrès " dans les mœurs, allant en définitive vers une rationalisation des pratiques - nous voulons dire: des pratiques "rationnelles quant aux fins", celles d'un pouvoir assez fort et d'une légitimité aux assises suffisamment affermies pour que son détenteur n'ait pas à se soumettre à la règle que nous serions portés à juger non seulement barbare (sommes-nous, Occidentaux, si bons juges en matière de barbarie ?) mais absurde de son élimination sacrificielle à échéance régulière. J'ajouterai que nous sommes en droit de penser qu'en employant ainsi les mots d'absurde et de barbare, nous nous laissons aller, en l'occurrence, à un processus d'identification imaginaire reposant, à vrai dire, sur une complète dénégation de l'altérité foncière de la personne du roi ou du chef sacré.

La société évhé n'est pas moins complexe ou moins "évoluée " que la société mossi. Nous n'avons pas, à dire vrai, de critère objectif pour apprécier le degré de complexité des sociétés que nous comparons sous un aspect déterminé. Pas plus pouvons-nous évaluer avec précision l'effectivité du pouvoir exercé par tel roi dans tel système politique.

26. Je renvoie au chapitre VIII de L'Odyssée du pouvoir, intitulé : "Mises à mort symboliques » (1992: 117). Celles-ci ont pour victimes, d'une part, l'un des jeunes fils du roi qui vient de mourir ; il porte le titre de kurita («mort régnant»), il est l'une de ses doublures qui est vouée alors à l'exil définitif. Le premier cheval du roi, d'autre part, qui sera lui effectivement tué sur les lieux de l'intronisation du successeur par ceux-là mêmes qui ont en charge la cérémonie du sacre. 
Mais j'ai toute raison de penser que le pouvoir du roi moundang (gô-Lere) ou du roi shilluk (reth) est tout à fait réel même s'il est, dans une certaine mesure, et c'est aussi le cas du roi mossi du Yatenga (yatenga-naba) comme de la plupart des souverains africains ${ }^{27}$, limité. Limité parce que partagé avec des conseils de dignitaires ou des serviteurs de cour qui sont - ou qui furent - bien autre chose que de simples exécutants sans volonté qui leur soit propre ${ }^{28}$.

Je terminerai en rappelant qu'à cet égard les analyses de Michel Izard, grand lecteur de Hegel, portent toutes la marque de la pensée dialectique qui est proprement la sienne tout au long de son travail sur le Yatenga. Bien avant la rédaction de Moogo qui en garde intacte l'inspiration, il souligne dans les pages de conclusion de Gens du pouvoir, gens de la terre, combien le caractère étatique de ce royaume est resté problématique. J'entends par là le mouvement qui tend à l'achèvement d'une forme idéale des rapports entre l'organisation de la société "civile" et le pouvoir ou, pour le dire autrement, à une conformité à l'idéologie étatique qui est à son origine, qui lui a donné son impulsion.

"Car ce pouvoir absolu est un pouvoir lacunaire, et d'abord parce que, pensée du politique, il n'est pas pensée de l'économique et donc demeure dans l'enfermement abstrait du verbe [...]. Le contrôle de la production appartient effectivement aux détenteurs de l'autorité lignagère, au niveau de ces grandes unités de production et de consommation que sont les yiya (les maisons)".

On en revient ainsi au questionnement qui était au point de départ de cet article et qui portait sur les contradictions inhérentes à l'incorporation d'une société lignagère au sein d'un royaume organisé en un État unitaire.

27. Cette remarque se justifie par les nombreuses études précises et approfondies des royautés africaines qui ont été publiées au cours des dernières décennies et qui contredisent pour une large part la thèse bien connue de George P. Murdock (Africa. Its People and their Culture History, 1959) sur le "despotisme " - dont l'arbitraire et la cruauté sont la marque - des chefs et des rois qui serait le caractère distinctif des systèmes politiques du Continent noir. Il ne s'agit pas d'en nier l'existence dans certains cas : comment, pour ne citer que celui dont il a été déjà question dans ces pages, ne pas penser à la manière dont les rois zulu, à commencer par Shaka, le premier et le plus fameux d'entre eux, traitaient les sujets récalcitrants. Mais, plus généralement - et il faut en parler au passé -, la sévérité dans la répression et parfois l'extrême cruauté dans l'exercice du pouvoir n'étaient pas le fait de l'arbitraire pur du despote, mais tenaient aux représentations et aux croyances partagées par le corps social, notamment mais pas seulement, à celles touchant à la sorcellerie et aux moyens de la combattre tout en l'utilisant. Je me permets de renvoyer le lecteur à mon livre Roi sorcier, mère sorcière (2006).

28. On ne peut pas ne pas penser à cette phrase que Fénelon, dans son Télémaque, met dans la bouche d'un sage que l'on veut faire roi malgré lui : "si la servitude est misérable, la royauté ne l'est pas moins, puisqu'elle est une servitude déguisée. Quand on est roi, disait-il, on dépend de tous ceux dont on a besoin pour se faire obéir ". 
«L'État, le lignage: entre les deux une solution de continuité [...]. Le pouvoir royal ne parvient pas, au travers de l'engendrement même des pouvoirs locaux, à pénétrer profondément l'univers des lignages; ou plutôt, il n'y parvient qu'au moyen d'un détour par la sacralité de la terre, où seul finalement est en cause le pouvoir royal, non pas d'ailleurs comme instance du pouvoir politique supérieur mais en tant qu'il s'incarne dans un roi, figure hors système [c'est moi qui souligne] qui renvoie à la notion totalisante de souveraineté ».

Précisons que le roi est évidemment «hors système " s'agissant du moos buudu, le lignage maximal issu de Wedraogo, le héros fondateur de la dynastie régnante comme il est, à plus forte raison, délié de toute dépendance vis-à-vis des normes régissant l'organisation lignagère qui est celle de l'ensemble de la société moose. Son statut le place à la frontière du monde social et du monde de la brousse commandé par les forces de la nature et les puissances invisibles dont dépend le destin des humains. Michel Izard poursuit :

"Le voilà bien l'imaginaire du pouvoir; ce roi-homme, ce royaume-femme, cette union de Wende et de Tenga, du ciel et de la terre. Mais où est, pratiquement, le lieu d'application de ce pouvoir ? [...] [L]e pouvoir ne pèse que sur les gens du pouvoir [...]. Mais le négatif de ce système hante ce monde toujours inachevé. Derrière le chef et le serviteur se profile toujours la "crapule", l' "homme qui se fout de tout"; derrière le sujet apparaît la silhouette du "malin"; et au-delà de ces deux figures bien réelles, l'une en retard sur l'histoire, l'autre peut-être en avance sur elle, celle du souverain, grand Un pétri d'imaginaire, vide sens : un symbole».

La société est bien pour l'État mais il reviendrait au même de dire qu'elle est contre, car que ce soit sous l'effet de la fascination par le Grand Un ou par soumission à la manifestation d'une force jugée invincible à ce moment de son histoire où pour elle un ordre nouveau lui a été imposé, la société a fini par accepter la loi de l'État comme une loi naturelle qui pour toujours lui reste extérieure. Mais, comme le disent très bien les dernières lignes que nous venons de citer de Gens du pouvoir, gens de la terre, l'État, dans sa complexité institutionnelle, peut aussi avoir affaire avec et contre lui-même, et peiner à dominer le conflit intérieur qui oppose la figure solaire de la souveraineté à celle, ténébreuse, de la force. 
Adler, Alfred

2006 Roi sorcier, mère sorcière. Parenté, politique et sorcellerie en Afrique noire. Paris, Le Félin-Kiron.

2010 «Logique sacrificielle et ordre politique : le statut de la personne du chef en relation avec son statut de sacrifiant ", Incidence 6 : 149-168.

\section{Apter, Andrew}

1992 Black Critics and Kings. The Hermeneutics of Power in Yoruba Society. Chicago, University of Chicago Press.

\section{Cartry, Michel}

1987 "Le suaire du chef ", in Michel Cartry, ed., Sous le masque de l'animal. Paris, Presses universitaires de France : 131-232.

\section{Desclés, Jean-Pierre}

2011 «La frontière et son concept ", communication orale.

\section{Evans-Pritchard, Edward E.}

1964 [1940] «Les Nouer du Soudan méridional ", in Edward Evans-Pritchard \& Meyer Fortes, eds, Systèmes politiques africains. Paris, Presses universitaires de France : 235-256.

\section{Fortes, Meyer}

1936 "Ritual Festivals and Social Cohesion in the Hinterland of the Gold Coast ", American Anthropologist 38 (4) : 590-604.

1964 [1940] «Le système politique des Tallensi des territoires au Nord de la Côte de l'Or ", in Edward Evans-Pritchard \& Meyer Fortes, eds, Systèmes politiques africains. Paris, Presses universitaires de France : 203-233.

\section{Hilgers, Mathieu}

2009 «Autodétermination et réflexivité dans les sociétés africaines anciennes (le cas moago) ", Journal des africanistes 78 (1-2) : 269-292.

\section{Horton, Robin}

1971 "Stateless Societies in the History of West Africa", in J. F. A. Ajayi \& Michael Crowder, eds, History of West Africa, 1. London, Longman : 78-119.

Izard, Michel

1982 «La politique extérieure d'un royaume africain : le Yatenga au XIX siècle ", Cahiers d'études africaines 87-88: 363-385.

1985a Gens du pouvoir, gens de la terre. Les institutions politiques de l'ancien royaume du Yatenga (bassin de la Haute-Volta blanche). Paris, Éd. de la Maison des sciences de l'Homme / Cambridge, Cambridge University Press.

1985b Le Yatenga précolonial. Un ancien royaume du Burkina. Paris, Karthala.

1986 "Le calendrier du Yatenga", Systèmes de pensée en Afrique noire 7 : 45-55.

1992 L'Odyssée du pouvoir. Un royaume africain: Etat, société, destin individuel. Paris, Éd. de l'École des hautes études en sciences sociales.

1995 «Une trifonctionnalité africaine? Représentations de la souveraineté et catégories socio-fonctionnelles dans le Yatenga ", Cahiers des sciences humaines 31 (2) : 407-425.

2003 Moogo. L'émergence d'un espace étatique ouest-africain au XVI siècle. Études d'anthropologie historique. Paris, Karthala.

Maine, Henry James Sumner

1861 Ancient Law. London, John Murray.

Maupoil, Bernard

1988 La Géomancie à l'ancienne Côte des Esclaves. Paris, Institut d'ethnologie.

Morgan, Lewis Henry

1877 Ancient Society. New York, Holt. 


\section{Murdock, George Peter}

1959 Africa. Its Peoples and their Culture History. New York, McGraw-Hill.

Radcliffe-Brown, Alfred R.

1964 [1940] "Préface », in Edward

E. Evans-Pritchard \& Meyer Fortes, eds, Systèmes politiques africains. Paris, Presses universitaires de France : XIII-XXIII.
Smith, Michael G.

1970 Government in Zazzau, 1800-1950.

London, Oxford University Press.

Surgy, Albert de

1990 «Le prêtre-roi des Evhés

du Sud-Togo ", Systèmes de pensée

en Afrique noire 10 : 93-120. 